\title{
DEANE CEDRAZ CARNEIRO
}

Início e dose de terapia renal substitutiva em pacientes com injúria renal aguda: avaliação de fatores prognósticos

Dissertação apresentada à Faculdade de Medicina da Universidade de São Paulo para obtenção do título de Mestre em Ciências

Programa de Nefrologia

Orientadora: Profa. Dra. Etienne Maria Vasconcellos de Macedo

\section{SÃO PAULO}


Dados Internacionais de Catalogação na Publicação (CIP)

Preparada pela Biblioteca da

Faculdade de Medicina da Universidade de São Paulo

Creprodução autorizada pelo autor

\section{Carneiro, Deane Cedraz}

Início de terapia renal substitutiva em pacientes com injúria renal aguda :

avaliação de fatores prognósticos / Deane Cedraz Carneiro. -- São Paulo, 2014.

Dissertação(mestrado)--Faculdade de Medicina da Universidade de São Paulo. Programa de Nefrologia.

Orientadora: Etienne Maria Vasconcellos de Macedo.

Descritores: 1.Diálise 2.Mortalidade 3.Epidemiologia 4.Oliguria 5.Creatinina 6.Índice de gravidade de doença

USP/FM/DBD-456/14 
Trabalho realizado no Departamento de Nefrologia, da Faculdade de Medicina da Universidade de São Paulo, com apoio financeiro do CNPq (Conselho Nacional de Desenvolvimento Científico e Tecnológico) pela concessão de bolsa de estudo (Proc.: 160757/2011-8). 


\section{Dedicatória}

Aos meus amores, Sebastien Brunel e Sophie Cedraz Brunel. Tudo em benefício da nossa família. 


\section{AGRADECIMENTOS}

A minha família amada, minha mãe Terezinha, meus irmãos, cunhados e sobrinhos, que mesmo longe não cansam de me incentivar. Verdadeiramente meu porto seguro.

A minha orientadora e amiga, Dra. Etienne Macedo, exemplo de profissionalismo e amor à pesquisa. Sempre preocupada com meu aprendizado, generosa ao compartilhar seus conhecimentos. Palavras de incentivo e suporte contínuo me fizeram descobrir competências que eu nem sabia que tinha. Quanta sorte eu tenho por ter sido sua orientanda!

A minha amiga querida, Dra. Andréa Magalhães, que tanto ajuda e incentiva minha carreira médica desde o início da residência em nefrologia. Sua alegria, sabedoria e dinamismo profissional são fontes de inspiração para mim.

Aos novos amigos que fiz nestes anos de pesquisa, Dra. Janaína Garcia e Dr. Maurício Teixeira, com quem dividi minhas angústias e com quem pude contar no momento que mais precisei. Tenho certeza que seremos amigos pra vida toda.

A Deus, fiel, que nunca me desampara. 
Esta dissertação está de acordo com as seguintes normas, em vigor no momento desta publicação:

Referências: adaptado de International Committee of Medical Journals Editors (Vancouver).

Universidade de São Paulo. Faculdade de Medicina. Divisão de Biblioteca e Documentação. Guia de apresentação de dissertações, teses e monografias. Elaborado por Anneliese Carneiro da Cunha, Maria Julia de A. L. Freddi, Maria F. Crestana, Marinalva de Souza Aragão, Suely Campos Cardoso, Valéria Vilhena. 3a ed. São Paulo: Divisão de Biblioteca e Documentação; 2011.

Abreviaturas dos títulos dos periódicos de acordo com List of Journals Indexed in Index Medicus. 


\section{SUMÁRIO}

Lista de Abreviaturas e Siglas

Resumo

Summary

1. INTRODUÇÃO

01

2. OBJETIVOS

12

3. MÉTODOS

3.1. Definições

3.2. Pacientes

3.2.1. Critérios de inclusão

3.2.1. Critérios de exclusão

3.3. Coleta de dados

3.4. Variáveis analisadas 18

3.5. Desfechos 22

3.6. Análise estatística e cálculo da amostra

3.6.1. Cálculo da amostra

3.6.2. Análise estatística

4.1 Características Clínicas

4.2 Parâmetros clínicos e laboratoriais no momento do início da TRS

4.3 Características da TRS

4.4 Evolução de parâmetros clínicos e laboratoriais antes e após início da TRS

4.5 Controle metabólico e do balanço hídrico após início da TRS

4.6 Características clínicas, laboratoriais e da TRS em sobreviventes e não sobreviventes

4.7 Parâmetros para início da TRS e mortalidade

4.8 Recuperação da função renal em relação às características clínicas, laboratoriais e da TRS

4.9 Recuperação da função renal em relação ao momento de início da 45 TRS 
5. DISCUSSÃO

6. CONCLUSÃO

7. ANEXOS

7.1 Anexo A

7.2 Anexо B

8. REFERÊECIAS BIBLIOGRÁFICAS 


\section{LISTA DE ABREVIATURAS E SIGLAS}

\begin{tabular}{|c|c|}
\hline AINES & Anti-inflamatórios não esteróides \\
\hline AKIN & Acute Kidney Injury Network \\
\hline APACHE II & Acute Physiology and Chronic Health Disease Classification System \\
\hline BUN & Blood urea nitrogen \\
\hline CAPPesq & Comissão de ética para análise de projetos de pesquisa do HCFMUSP \\
\hline CEC & Circulação extracorpórea \\
\hline CVVHDF & Hemodiafiltração veno-venosa contínua \\
\hline DPOC & Doença pulmonar obstrutiva crônica \\
\hline DRC & Doença renal crônica \\
\hline DVA & Droga vasoativa \\
\hline EUA & Estados Unidos da América \\
\hline HAS & Hipertensão arterial sistêmica \\
\hline \multirow[t]{2}{*}{ HCFMUSP } & Hospital das clínicas da faculdade de medicina da universidade de São \\
\hline & Paulo \\
\hline ICC & Insuficiência cardíaca \\
\hline IECA & Inibidores da enzima conversora de angiotensina \\
\hline IRA & Injúria renal aguda \\
\hline MDRD & Modification of Diet in Renal Diseases \\
\hline PAM & Pressão arterial média \\
\hline PCR & Proteína $\mathrm{C}$ reativa \\
\hline
\end{tabular}


RIFLE Risk, Injury, Failure, Loss and End-Stage Kidney Disease

SAPS 3 Simplified Acute Physiology Score

SLED Sustained Low-Efficiency Dialysis

SOFA Sequencial Organ Failure Assessment

$\mathrm{sCr} \quad$ Creatinina sérica

TRS Terapia renal substitutiva

UTI Unidade de terapia intensiva

VM Ventilação mecânica

VNI Ventilação não invasiva

vs. $\quad$ Versus 


\section{RESUMO}

Carneiro DC. Início e dose de terapia renal substitutiva em pacientes com injúria renal aguda: avaliação de fatores prognósticos. [dissertação]. São Paulo: Faculdade de Medicina da Universidade de São Paulo; 2014.

INTRODUÇÃO: A despeito do avanço no conhecimento fisiopatológico da injúria renal aguda (IRA), da sofisticação dos métodos de substituição renal e do suporte de pacientes em terapia intensiva, a mortalidade associada à IRA continua elevada. Dessa forma, melhorias na prática de diálise, como início precoce da TRS e aumento da dose de diálise, permanecem como assuntos de intenso debate. Este trabalho foi planejado com intuito de caracterizar o perfil clínico e laboratorial dos pacientes no início da terapia dialítica, e estudar a influência do tempo de início e da dose de diálise na evolução dos pacientes com IRA em UTI. O objetivo principal foi comparar a mortalidade hospitalar em relação ao momento de início da TRS de acordo com diferentes parâmetros. MÉTODOS: Estudo prospectivo de coorte observacional, onde foram analisados dados clínicos e laboratoriais de pacientes internados em UTI. O momento de início da TRS foi definido de acordo com o nível de uréia (precoce se menor que $120 \mathrm{mg} / \mathrm{dL}$ ), estágio do AKIN (precoce se TRS com AKIN 1 ou 2) e tempo de internação em UTI no início da TRS (precoce quando TRS menor que 2 dias). A recuperação renal completa foi definida como relação $\mathrm{sCr}$ alta/sCr basal menor ou igual a $20 \%$, e recuperação parcial a relação maior que $20 \%$, mas sem dependência de TRS. RESULTADOS: Foram analisados dados de 168 pacientes. A mediana da idade dos pacientes foi 55 anos. As medianas dos escores de gravidade no momento do início da diálise foram 74 (67-82) e 14 (11-17) para SAPS 3 e SOFA, respectivamente. A mortalidade hospitalar dos pacientes submetidos à TRS foi 73,2\%. Os pacientes que não sobreviveram tinham SAPS 3 maior que os pacientes sobreviventes ( $75 v s .67, \mathrm{p}=0,041)$. O balanço hídrico acumulado, no início da TRS, foi maior nos pacientes que evoluíram para óbito quando comparados com os pacientes sobreviventes $(3090 \mathrm{ml} v s .1632 \mathrm{ml}, \mathrm{p}=0,004)$. A dose de diálise não apresentou relação com a mortalidade hospitalar. Não houve diferença em relação à mortalidade hospitalar quando o nível de uréia no início da TRS era maior que 120 $\mathrm{mg} / \mathrm{dL}$ comparado com uréia menor que $120 \mathrm{mg} / \mathrm{dl}$. A mortalidade hospitalar entre os pacientes que iniciaram a terapia dialítica com AKIN 3, comparados com os pacientes que iniciaram com AKIN 1 ou 2 também não foi diferente. Em relação ao tempo de internação em UTI no início da TRS, pacientes que iniciaram a TRS antes de 2 dias da internação tiveram menor mortalidade hospitalar, 62,7\% vs. 73,9\% quando a TRS foi iniciada entre 2 dias e 5 dias, vs. 82,5\% quando TRS iniciada após 5 dias de admissão em UTI; $p=0,046$. Na análise multivariada, tempo de internação em UTI no início da TRS manteve associação com mortalidade hospitalar (OR 4,70; 95\% IC 1,0-21,9 para os pacientes que começaram entre 2-5 dias e OR 20,93; 95\% IC 3,1-139,3 para os pacientes que começaram após 5 dias de admissão na UTI). Análise do tempo de início e dose de diálise em relação à recuperação da função renal, não foi diferente quando comparado o grupo que recuperou a função renal total com o grupo que recuperou parcialmente. CONCLUSÃO: O início precoce baseado em dados laboratoriais relacionados à gravidade da IRA, nível de uréia e o estágio da 
IRA baseado na classificação de AKIN, no início da TRS, não foram associados com a mortalidade hospitalar. O início precoce em relação ao tempo de internação em UTI no início da TRS esteve independentemente associado a menor mortalidade hospitalar. $\mathrm{O}$ atraso no reconhecimento da gravidade da IRA, assim como para a instituição do tratamento dialítico, pode ter impacto na evolução dos pacientes com IRA. Estudos futuros devem avaliar se o início precoce, baseado em parâmetros temporais, pode ser um fator que altere a mortalidade associada à IRA dialítica.

Descritores: Diálise, mortalidade, epidemiologia, oligúria, creatinina, índice de gravidade de doença. 


\title{
SUMMARY
}

\author{
CARNEIRO DC. Timming and dose of renal replacement therapy in patients with \\ acute kidney injury: evaluation of prognostic factors. [dissertation]. São Paulo: \\ "Faculdade de Medicina, Universidade de São Paulo"; 2014.
}

BACKGROUND: Despite recent advance on acute kidney injury (AKI) diagnosis and the sophistication of the methods of renal replacement and support, the mortality associated with AKI remains high. Thus, improvement in the practice of RRT for AKI patients, such as early onset and adequate dose, remain issues of intense debate. The aim of this study is to characterize the clinical and laboratory profiles of patients before and at RRT initiation and assess the effect of the timing of RRT initiation on outcomes of patients with AKI in ICU. The main objective is to compare mortality in relation to the RRT initiation according to different parameters. METHODS: This is a prospective observational cohort study. Clinical and laboratory data of patients in the ICU were analyzed. Early and late start was defined according to the level of urea (early if less than $120 \mathrm{mg} / \mathrm{dL}$ ), AKIN stage (early if RRT with AKIN 1 or 2) and time from ICU admission to RRT initiation (RRT early as less than 2 days). Complete recovery was defined as the ratio of $\mathrm{sCr}$ hospital discharge / baseline $\mathrm{sCr}$ less than or equal to $20 \%$ and partial recovery as a ratio higher than $20 \%$, but without the need for RRT. RESULTS: 168 patients were analyzed. The median age was 55 years. The median severity scores at the initiation of dialysis was 74 (67-82) and 14 (11-17) for SAPS 3 and SOFA, respectively. Hospital mortality of patients undergoing RRT was $73.2 \%$. Non-survivors patients had greater SAPS III than survivors patients $(75$ vs $67, \mathrm{p}=0.041)$. The cumulative fluid balance at the beginning of the RRT was higher in non-survivors compared to survivors (3090 ml vs. $1632 \mathrm{ml}, \mathrm{p}=0.004)$.The dialysis dose was not associated with mortality. There was no difference in mortality when the urea level was greater than $120 \mathrm{mg} / \mathrm{dL}$ at RRT initiation compared to urea less than $120 \mathrm{mg} / \mathrm{dl}$. There was no difference inhospital mortality among patients who started dialysis with AKIN 3 when compared with patients who started with AKIN 1 or 2 . Time from ICU admission to RRT initiation was associated with lower hospital mortality $(62.7 \%$ when the RRT started before 2 days vs. $73.9 \%$ when the RRT started between 2 days and 5 days vs. $82.5 \%$ when RRT started after 5 days of admission to the ICU; $p=0.046$ ). In the multivariate analysis, time from ICU admission to RRT initiation remained significantly associated with hospital mortality (OR 4.70; 95\% CI 1.0 to 21.9 for those starting from 2-5 days; and OR 20.93; $95 \%$ CI 3.1 to 139.3 for patients starting with more than 5 days). The timing of beginning and the dialysis dose were not different in the group of patients that completely recovered renal function as compared to the group with partial recovery. CONCLUSION: Early start based on laboratorial data associated with AKI severity, urea level or AKIN stage, was not associated with decreased mortality. Early start based on time from ICU admission to RRT initiation was independently associated with lower mortality. The delay in AKI severity recognition and to start RRT can impact outcome of AKI patients. Future studies should investigate whether an early start could impact AKI mortality. 
Descriptors: Dialysis, mortality, epidemiology, oliguria, creatinine, severity of illness index. 


\section{INTRODUÇÃO}

Nas últimas décadas, a despeito do avanço no conhecimento fisiopatológico da injúria renal aguda (IRA), da sofisticação dos métodos de substituição renal e do suporte de pacientes em terapia intensiva, a mortalidade associada à IRA continua elevada. Apesar de estudos mais recentes mostrarem que a mortalidade diminuiu (14), ela persiste alta: 40 a $70 \%(5,6)$. O maior número de fatores contribuindo para a IRA, como o aumento de intervenções terapêuticas e diagnósticas e a presença de disfunção de múltiplos órgãos em pacientes mais velhos, colaboram para manter as altas taxas de mortalidade (7).

Desde 2004 após publicação do RIFLE (8) e em 2007 após publicação dos novos critérios para diagnóstico e classificação da IRA, o AKIN (acrônimo em inglês para o critério proposto pelo “Acute Kidney Injury Network”), (9) minimizou-se a heterogeneidade na definição da IRA apresentada nos trabalhos, o que dificultava a comparação entre os estudos e limitava o entendimento desta síndrome (10-12). Obedecendo a esses critérios, passou-se a definir IRA como um aumento da creatinina sérica $(\mathrm{sCr})$ de $0,3 \mathrm{mg} / \mathrm{dL}$ ou aumento de 1,5 vezes na $\mathrm{sCr}$ em relação a referência em $48 \mathrm{~h}$ ou uma diurese $\leq 0,5 \mathrm{~mL} / \mathrm{Kg} /$ hora por $6 \mathrm{~h}$. A classificação proposta pelo KDIGO ("Kidney disease improving global outcomes”) é também baseada neste critério, apenas com uma mudança adicional (13). Na mudança proposta, o aumento de 1,5 vezes na $\mathrm{sCr}$ passou a ser avaliado em sete dias. Assim, a IRA ficou dividida em três estágios como se segue: 
Estágio 1: aumento em 1,5 - 1,9 vezes da $\mathrm{sCr}$ ou aumento de $\geq 0,3 \mathrm{mg} / \mathrm{dL}$; e/ou débito urinário < 0,5 ml/kg/h em 6 a 12h;

Estágio 2: aumento em 2,0 - 2,9 vezes da sCr; e/ou débito urinário $<0,5$ $\mathrm{ml} / \mathrm{kg} / \mathrm{h} \mathrm{em} \geq 12 \mathrm{~h} ;$

Estágio 3: aumento em 3,0 vezes da $\mathrm{sCr}$ ou aumento da $\mathrm{sCr}$ para $\geq 4,0 \mathrm{mg} / \mathrm{dL}$ ou início da terapia renal substitutiva (TRS) ou queda na taxa de filtração glomerular estimada para $<35 \mathrm{ml} / \mathrm{min}$ por $1,73 \mathrm{~m}^{2}$ em pacientes < 18 anos; e/ou débito urinário $<0,3 \mathrm{ml} / \mathrm{kg} / \mathrm{h}$ em $\geq 24 \mathrm{~h}$ ou anúria por $\geq 12 \mathrm{~h}$.

Nos últimos anos, os avanços na medicina intensiva e na tecnologia em diálise, como o uso de membranas biocompatíveis de alto fluxo, terapias contínuas, proporcionaram melhorias no cuidado geral dos pacientes críticos com IRA, resultando na diminuição da mortalidade (1). Contudo, a mortalidade na IRA permanece bastante elevada e por isso, mudanças nas práticas de diálise, como o momento ideal para iniciar a TRS e a dose de diálise necessária para melhorar desfechos clínicos em pacientes críticos, permanecem como assuntos de intenso debate.

O reconhecimento de que a morbidade e mortalidade estão inversamente relacionadas à dose de diálise em pacientes com doença renal crônica (DRC) gerou mudanças nas práticas de diálise. Desde 1976 o estudo multicêntrico NCDS (National Cooperative Dialysis Study) avaliou a relação entre a dose oferecida de diálise e a evolução dos pacientes renais crônicos. O estudo demonstrou que, pacientes com maior remoção de solutos, medido pela concentração de uréia no tempo, apresentavam menor morbidade. A associação sugere que a quantidade de 
solutos de baixo peso molecular removidos, está associada à mortalidade e, portanto, a medida da cinética da uréia deveria ser usada para adequar o tratamento hemodialítico $(14,15)$. O Kt/V é o índice utilizado para quantificação da dose de diálise oferecida a pacientes com DRC. Nesse índice, o K representa a depuração de uréia através do dialisador $(\mathrm{mL} / \mathrm{min})$, t, o tempo de diálise $(\min ), \mathrm{e} \mathrm{V}$, o volume de distribuição de uréia no corpo $(\mathrm{mL})$. Considera-se adequado um valor de $\mathrm{Kt} / \mathrm{V}=1,2$ quando se utiliza o modelo de cinética da uréia de único compartimento.

O modelo de cinética da uréia utilizado em DRC foi extrapolado, e é, atualmente, também utilizado para pacientes com IRA. Além das críticas da utilização desse modelo matemático em pacientes que estão em balanço nitrogenado negativo e com variação do volume corporal total, o Kt/V prescrito aos pacientes é frequentemente não atingido. Vários são os fatores relacionados ao procedimento e relacionados ao paciente, que contribuem para essa diferença entre dose prescrita e oferecida. A depuração através do dialisador é afetada pelo fluxo de sangue e de dialisato, pela qualidade e recirculação do acesso vascular e pelas características da membrana. Em decorrência dos frequentes episódios de hipotensão e problemas com o acesso vascular, o fluxo de sangue em hemodiálise nos pacientes com IRA ou mesmo nos pacientes com DRC internados em UTI é, na grande maioria, menor do que nos pacientes crônicos estáveis. A performance do dialisador e a eficiência da diálise são também influenciadas pela anticoagulação, muitas vezes reduzida ou não utilizada nos pacientes em UTI devido às coagulopatias e à presença de sangramentos. A recirculação através do cateter de diálise também é um problema: os cateteres temporários podem apresentar recirculação de até $26 \%$, prejudicando a eficiência da diálise. O tempo de hemodiálise nesses pacientes também é 
frequentemente reduzido por interrupção precoce devido à coagulação do dialisador, episódios de hipotensão ou necessidade de realização de procedimentos diagnósticos ou terapêuticos.

A dose de diálise oferecida é, em geral, medida através de fórmulas que não medem diretamente a massa de uréia extraída e utilizam somente a medida de uréia pré e pós-diálise. Essas fórmulas se baseiam em uma cinética de uréia que supõe sua distribuição por um compartimento único, geração e produção em equilíbrio e volume de distribuição constante. Dessa forma, a queda da concentração de uréia no sangue, produzida pela hemodiálise, é considerada como a remoção de uréia por todos os tecidos. O reequilíbrio da uréia entre os tecidos e o compartimento intravascular ocorre em 30 a 60 minutos após término da sessão de hemodiálise, e a diferença entre a concentração plasmática de uréia imediatamente após o término da diálise e a concentração obtida 30 a 60 minutos após é tanto maior quanto maior é a eficiência do método. Esse efeito rebote é ainda mais evidente quando ocorrem períodos de hipotensão e/ou instabilidade hemodinâmica durante a diálise (16). O chamado efeito rebote da uréia mostra que a remoção da uréia entre os diferentes compartimentos não é constante durante o procedimento dialítico.

A dose, bem como o método mais adequado de diálise, oferecida a pacientes com IRA foram foco de vários estudos retrospectivos e prospectivos nas últimas duas décadas. A definição de diálise adequada em IRA é complexa e envolve, além do método utilizado, o controle metabólico, hidroeletrolítico e hemodinâmico do paciente (17). Um dos primeiros estudos retrospectivos que chamou atenção para dose de diálise em pacientes com IRA foi publicado por Paganini e col. (18). Os autores avaliaram a evolução de 842 pacientes, que necessitaram de hemodiálise 
intermitente ou terapias contínuas, baseadas no escore de gravidade da Cleveland Clinic. Para os pacientes com escores muito baixos ou muito altos, a dose de diálise oferecida não teve influência. No entanto, nos pacientes com escores intermediários o uso da terapia contínua permitiu que recebessem maior dose de diálise com menor instabilidade hemodinâmica. Ronco e col. (19) randomizaram 425 pacientes com IRA em UTI para avaliar o impacto na mortalidade de doses crescentes de hemofiltração. Foram utilizadas três doses de hemofiltração: ultrafiltração de 20 $\mathrm{mL} / \mathrm{Kg} / \mathrm{h}$ (grupo 1, n=146), $35 \mathrm{~mL} / \mathrm{Kg} / \mathrm{h}$ (grupo 2, n=139) ou $45 \mathrm{~mL} / \mathrm{Kg} / \mathrm{h}$ (grupo 3, $\mathrm{n}=140)$. A sobrevida no grupo $1(41 \%)$ foi significantemente menor que nos grupos 2 (57\%) e $3(58 \%)$, mostrando que o aumento na dose de diálise (hemofiltração) esteve relacionado a maior sobrevida.

Schiffl e col. (20) dialisaram 160 pacientes com IRA em UTI utilizando hemodiálise intermitente em dias alternados ou diariamente. Os pacientes eram semelhantes quanto à idade, sexo, gravidade e causa da IRA. A hemodiálise diária resultou em melhor controle da uremia, menos episódios de hipotensão durante a diálise ( $5 \pm 2 \%$ vs. $25 \pm 5 \%, \mathrm{P}<0,001)$, maior dose de diálise (Kt/V semanal 5,8 $\pm 0,4$ vs. 3,0 $\pm 0,6)$ e resolução mais rápida da IRA $(9 \pm 2$ vs. $16 \pm 6$ dias, $\mathrm{P}<0,001)$. A mortalidade foi de $28 \%$ no grupo dialisado diariamente e $46 \%$ no grupo dialisado em dias alternados $(\mathrm{p}<0,001)$. A crítica mais importante a esse trabalho está no critério de inclusão dos pacientes, pois aqueles que tinham indicação de terapias contínuas de diálise foram excluídos, portanto foram estudados os pacientes de menor gravidade. Este fato se reflete na mortalidade encontrada que foi muito baixa, mesmo no grupo dialisado em dias alternados, quando comparada à mortalidade descrita na literatura para pacientes em UTI com IRA dependente de diálise. Mesmo assim, o trabalho 
mostra que, como nos pacientes com DRC, a dose de diálise pode estar relacionada à morbidade e mortalidade na IRA.

A heterogeneidade da população e dos tratamentos dialíticos nos pacientes internados em UTI tem mostrado a importância da quantificação da dose de diálise que é ofertada a estes pacientes. Além da dificuldade de comparar os métodos intermitentes e contínuos, não se pode considerar constante o volume de distribuição da uréia nesses pacientes e nem a sua geração. Até o momento não está estabelecido o melhor modo para se avaliar a dose de diálise oferecida aos pacientes críticos, com IRA ou mesmo com DRC. Idealmente outros parâmetros de avaliação da dose deveriam ser considerados como o controle metabólico, ácido básico e do balanço hídrico. Estes parâmetros avaliariam também a eficiência da TRS, já que a dose alta, por exemplo, não significaria necessariamente diálise eficiente.

Além da dose de diálise, outro fator determinante está relacionado ao momento de início da terapia dialítica. Apesar de não haver hesitação para iniciar o tratamento dialítico em casos de hipercalemia, hipervolemia e acidose refratária, na ausência dessas indicações clássicas, o momento de início de diálise é muito variável na prática clínica. Após os últimos dois grandes estudos clínicos multicêntricos avaliando dose de diálise, o VA/NIH Acute Renal Failure Trial Network (ATN) Study (21) nos EUA e o Randomized Evaluation of Normal versus Augmented Level of RRT (RENAL) (22) na Austrália, terem falhado em demonstrar diminuição da mortalidade em pacientes que receberam maiores doses de diálise, o possível benefício do início precoce da diálise passa a ser o próximo parâmetro a ser avaliado. 
Muitos fatores, incluindo logística, disponibilidade de recursos, experiência médica e fatores relacionados ao paciente estão envolvidos na decisão de quando começar a TRS para pacientes com IRA. Os fatores relacionados ao paciente incluem os níveis de creatinina e uréia, a gravidade da disfunção orgânica não renal, acúmulo de líquido e oligúria (23). Além dos fatores relacionados ao paciente, precisam ser levados em consideração os riscos de efeitos adversos associados com o procedimento dialítico que inclui hipotensão, sangramento, complicações relacionadas ao cateter, etc. Uma decisão por um início "precoce” da TRS num paciente com possibilidade de recuperar espontaneamente a função renal poderia expô-lo aos potenciais efeitos adversos da terapia dialítica de maneira desnecessária (24). A atual falta de consenso quanto aos benefícios da TRS "precoce" resulta na tendência que levam alguns médicos, no momento de indicar a TRS, dar um peso maior às conseqüências adversas do procedimento em detrimento aos potenciais benefícios da terapia dialítica (25-27).

Além disso, a TRS tem sido associada com taxas de mortalidade mais elevadas em comparação aos pacientes mantidos em tratamento conservador $(1,28$, 29). No entanto, existem várias diferenças nos padrões de gravidade entre os doentes submetidos à TRS e aqueles em que o tratamento conservador é preferido $(30,31)$. Assim, a associação de TRS e maiores taxas de mortalidade provavelmente são determinadas por outros fatores como a gravidade da doença, já que não existe consenso sobre as indicações para a TRS.

Em um estudo de coorte belga, incluindo nove unidades de cuidados intensivos, foi observado a evolucão de 1.303 pacientes com IRA ( $\mathrm{sCr}>2 \mathrm{mg} / \mathrm{dL}$ ), 
que receberam tratamento conservador ou TRS intermitente ou contínua (30). Em pacientes submetidos à TRS, houve maior mortalidade (58\% vs. $43 \%)$ e maior tempo de internação tanto na UTI quanto hospitalar, em relação aos que permaneceram em tratamento conservador. Foi utilizado o score SHARF (Stuivenberg Hospital Acute Renal Failure) para avaliar e ajustar para a gravidade dos pacientes. O risco de morte no grupo TRS em relação ao grupo tratamento conservador foi 1,75 (IC 95\%: 1,42,3). Após correção para a idade, tipo de IRA e escores de gravidade APACHE II (“Acute Physiology and Chronic Health Evaluation”) e SOFA (“Sequential Organ Failure Assessment”), foi confirmada maior mortalidade no grupo TRS. Este estudo levanta a hipótese que a mortalidade esperada em pacientes com IRA que recebem TRS não pode ser explicado somente pela maior gravidade da doença nos pacientes deste grupo.

Em recente análise de um estudo francês, multicêntrico e prospectivo, Clec `’h e colaboradores avaliaram a eficácia da TRS com atenção especial ao momento do início da TRS (32). Dos 2.846 pacientes, aqueles que receberam TRS (19\%) tiveram taxas de mortalidade mais elevadas do que os pacientes sem TRS (45,1\% vs. 23,4\%, $\mathrm{P}<0,001)$. Com o objetivo de aumentar a precisão desse resultado, foi utilizado um escore de propensão para controlar o viés de seleção de tratamento. Após ajuste para este escore, os pacientes que receberam TRS permaneciam com taxas de mortalidade mais elevadas que seus respectivos controles $(38,9 \%$ vs $22,2 \%, \mathrm{P}<0,001)$. Eles concluíram que, TRS não conseguiu reduzir a mortalidade hospitalar e enfatizou a necessidade de estudos randomizados que comparam terapia dialítica e tratamento conservador em pacientes de UTI, com especial enfoque no momento de início da TRS. Porém, utilizando também um escore de propensão, Libório e col.(33) 
mostraram que TRS foi associada com uma melhor sobrevida hospitalar (OR, 0,75; IC 95\%, 0,58-0,96), nos pacientes que apresentavam complicações relacionadas com a IRA (acidose metabólica, hipercalemia, sobrecarga hídrica).

Vários são os fatores que dificultam a avaliação do tempo de início de diálise como um parâmetro relacionado à evolução de pacientes com IRA. A maioria dos estudos que avaliaram o início da terapia dialítica foi retrospectiva. Entre eles, a maioria define o início como precoce ou tardio baseado em parâmetros laboratoriais como uréia e creatinina séricas. Dois estudos retrospectivos mostraram benefício do início precoce baseado em níveis de uréia sérica $(34,35)$. Em pacientes com trauma grave, a uréia $<120 \mathrm{mg} / \mathrm{dL}$ no início da terapia dialítica esteve associada a um risco absoluto na mortalidade de $20 \%$ (34). Na população geral de UTI, um grande estudo observacional mostrou aumento do risco de óbito quando a terapia dialítica foi iniciada com uréia > $150 \mathrm{mg} / \mathrm{dL}$ (35). Nessa coorte, o risco para óbito relacionado ao início tardio de terapia dialítica foi 1.85 (IC 95\%: 1.16-2.96), já ajustado para idade, falência hepática, sepse, plaquetopenia, creatinina sérica e modalidade terapêutica empregada.

Saudan e colaboradores, avaliando pacientes submetidos à hemodiafiltração veno-venosa continua (CVVHDF) (36), mostrou que os níveis de uréia no início do procedimento estavam independentemente associados à mortalidade em 28 e 90 dias. Em estudo realizado em um único centro, Bouman e colaboradores randomizaram 106 pacientes oligúricos para terapia de início precoce ou tardio (37). Apesar desse estudo não ter poder estatístico para responder a questão, não houve diferença na mortalidade dos dois grupos. 
Alguns estudos também basearam o tempo de início da terapia dialítica no diagnóstico de IRA e dias de internação em UTI. Cosentino e colaboradores (5), em uma coorte com 363 pacientes de terapia intensiva demonstrou que o tempo de início de diálise em relação ao tempo de internação na UTI estava independentemente relacionado a uma maior mortalidade. Esse parâmetro também foi avaliado no estudo do grupo BEST ("Beginning and Ending Supportive Therapy”) (38). Nesse estudo, os investigadores estratificaram os pacientes baseados em valores relativos e absolutos de creatinina e uréia e em relação ao tempo de internação na UTI. Pacientes com início da TRS em até dois dias da internação foi considerado precoce, entre dois e cinco dias atrasado e mais que cinco dias, tardio. O único parâmetro de início de dialise que foi independentemente relacionado com maior risco de óbito foi o tempo de internação na UTI.

Os dois últimos estudos multicêntricos avaliando dose de diálise, o VA/NIH Acute Renal Failure Trial Network (ATN) (21) Study e o Randomized Evaluation of Normal versus Augmented Level of RRT (RENAL) na Austrália (22), também avaliaram alguns parâmetros relacionados ao início da terapia dialítica. A média de BUN no início da terapia dialítica foi praticamente idêntica nos grupos randomizados para terapia intensiva e menos intensa $(65.9 \mathrm{mg} / \mathrm{dL}[23.5 \mathrm{mmol} / \mathrm{L}] v s .66 .7 \mathrm{mg} / \mathrm{dL}$ [23.8 mmol/L]). Nesses estudos, o tempo para início da terapia dialítica em relação à admissão na UTI não foi relacionado à mortalidade ou aos outros desfechos avaliados. No estudo ATN o tempo médio de início da terapia dialítica foi de seis dias nos dois grupos. 
Este trabalho foi planejado com intuito de caracterizar o perfil clínico e laboratorial dos pacientes no início da terapia dialítica, e estudar a influência do tempo de início e da dose de diálise, na evolução dos pacientes com IRA em UTI. 


\section{OBJETIVOS}

\subsection{Objetivo principal}

Avaliar se o momento de início da TRS, baseado em diferentes definições de precocidade, esta associado à mortalidade hospitalar em pacientes com injúria renal aguda submetidos à hemodiálise em unidade de terapia intensiva.

\subsection{Objetivos secundários}

2.2.1 Avaliar se momento de início da TRS, baseado em diferentes definições de precocidade, esta associado à recuperação da função renal.

2.2.2 Avaliar se os parâmetros clínicos e laboratoriais dos pacientes no momento do início da TRS estão associados com a mortalidade e recuperação da função renal.

2.2.3 Avaliar se o tempo para o controle metabólico e do balanço hídrico dos pacientes esta associado a mortalidade e à recuperação da função renal.

2.2.4 Avaliar se a dose de diálise oferecida durante o período de diálise esta associada a mortalidade e à recuperação da função renal. 


\section{MÉTODOS}

Este trabalho foi desenvolvido no Instituto Central do Hospital das Clínicas da Faculdade de Medicina da Universidade de São Paulo (HCFMUSP), um hospital universitário, público e terciário.

É um estudo prospectivo de coorte observacional, onde foram analisados dados clínicos e de exames laboratoriais de pacientes internados em UTI. Os dados laboratoriais e clínicos foram obtidos a partir de anotações em prontuários médicos, que fazem parte da rotina assistencial da equipe multiprofissional do setor de terapia intensiva. O executante não participa da equipe multiprofissional.

O Projeto foi aprovado pela comissão de ética para análise de projetos de pesquisa (CAPPesq) do HCFMUSP (registro on-line número 8162, protocolo CAPPesq número 0639/11).

\subsection{DEFINIÇÕES}

\section{Doença renal crônica}

Para determinação da presença de doença renal crônica (DRC) prévia, foi utilizado o menor valor da creatinina entre três e seis meses da internação. Para pacientes sem dosagens da creatinina neste período, foi utilizado o menor valor da sCr encontrada nos três meses antes da internação até sete dias após admissão hospitalar se este valor correspondesse a um MDRD ("Modification of Diet in Renal Disease Study") (39) calculado maior que $90 \mathrm{~mL} / \mathrm{min}$ por $1,73 \mathrm{~m}^{2}$. Feito isto, 
pacientes com MDRD menor que $60 \mathrm{~mL} / \mathrm{min}$ por $1,73 \mathrm{~m}^{2}$ foram considerados como portadores de DRC.

\section{Injúria Renal Aguda}

Diagnóstico e gravidade da IRA foi definido pelo sistema de classificação AKIN utilizando-se os critérios da creatinina ou diminuição do débito urinário. Para o cálculo do AKIN foi utilizado como creatinina basal, em ordem de preferência decrescente: 1 . O menor valor da $\mathrm{sCr}$ encontrado dos três aos seis meses anteriores a internação; 2. O menor valor da $\mathrm{sCr}$ encontrado dos três meses antes da internação até sete dias após admissão hospitalar.

\section{Recuperação da Função Renal}

Recuperação da função renal nos sobreviventes foi avaliada na alta da UTI e hospitalar através da razão da $\mathrm{sCr}$ da alta (UTI e/ou hospitalar) em relação à $\mathrm{sCr}$ basal. Foi definida como recuperação completa da função renal se a relação $\mathrm{sCr}$ alta/sCr basal foi menor ou igual a $20 \%$ e como recuperação parcial se a relação foi maior que 20\%, mas sem dependência de TRS (Figura 1). 


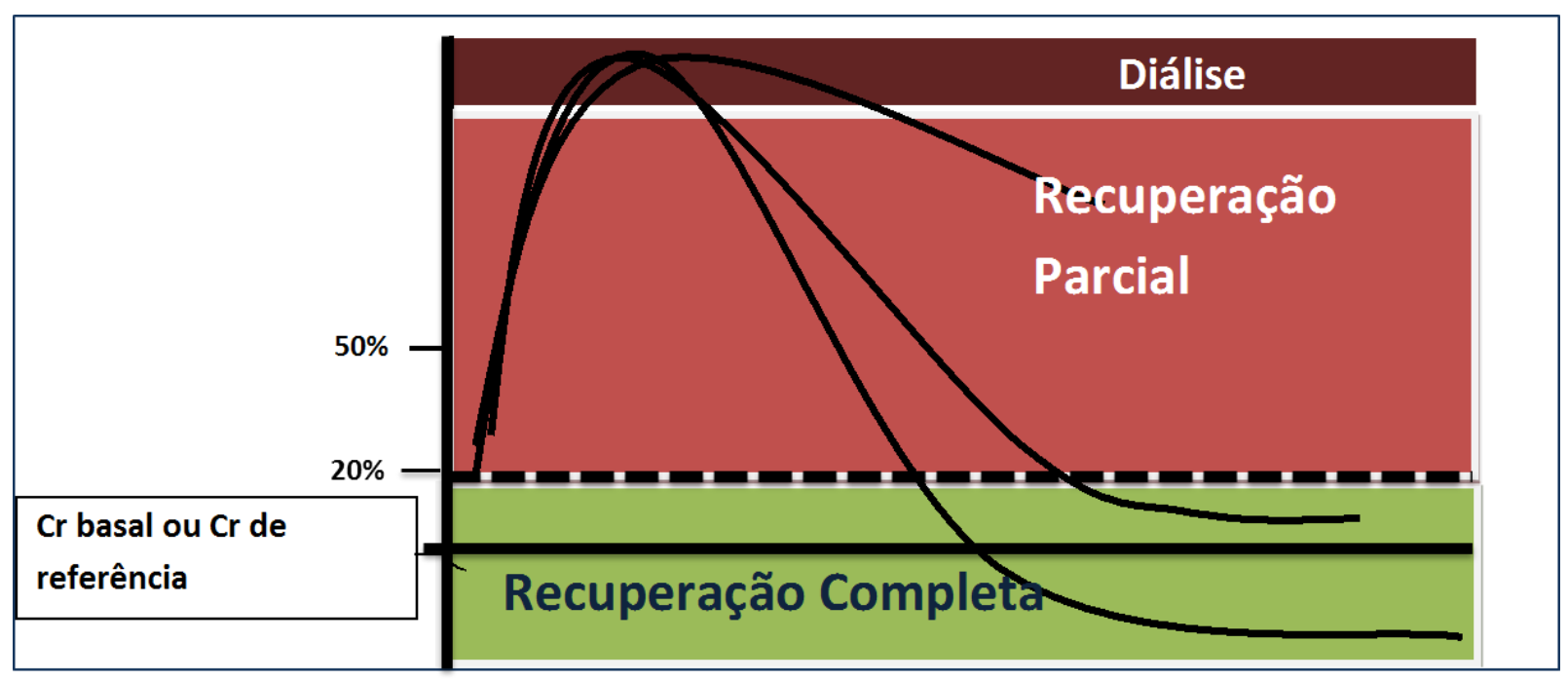

Figura 1 - Recuperação da função renal

Momento de início da TRS

O momento de início da TRS foi definido de acordo com o nível de uréia, estágio do AKIN e tempo de internação em UTI no início da TRS. O nível de uréia definido como precoce foi menor que $120 \mathrm{mg} / \mathrm{dL}$. Para classificação de AKIN foi utilizado os critérios de $\mathrm{sCr}$ e débito urinário. Pacientes iniciando a TRS com AKIN 1 ou 2 foram considerados como precoce e AKIN 3 como tardio. O tempo de internação em UTI no início da TRS menor que dois dias foi considerado como início precoce. 


\section{Controle metabólico e de balanço hídrico}

O controle metabólico foi baseado nos níveis de uréia, bicarbonato e $\mathrm{pH}$. Foi considerado que o paciente atingiu o controle metabólico da uréia quando, durante o período dialítico, a uréia atingiu valores menores que $100 \mathrm{mg} / \mathrm{dL}$. Foi considerado que o paciente atingiu o controle acidobásico quando, durante o período dialítico, o bicarbonato arterial e $\mathrm{pH}$ arterial séricos atingiram valores maiores que $22 \mathrm{mEq} / \mathrm{L} \mathrm{e}$ 7,35 , respectivamente.

O balanço hídrico $(\mathrm{BH})$ diário foi calculado como a soma dos ganhos e perdas de fluidos do paciente, não sendo descontadas perdas insensíveis, e o BH acumulado foi calculado como a soma dos balanços diários. Foi calculada ainda a porcentagem do $\mathrm{BH}$ em relação ao peso usando a seguinte fórmula: [BH acumulado / peso corpóreo $(\mathrm{kg})$ x 1000] x 100. Foi considerado que o paciente obteve o controle do balanço hídrico quando o $\mathrm{BH}$ acumulado atingiu valor igual ou menor que zero.

\subsection{PACIENTES}

Foi incluída no estudo uma amostra não probabilística, por conveniência, de pacientes criticamente enfermos, internados em UTIs clínicas e cirúrgicas do HCFMUSP. As UTIs cirúrgicas incluídas no estudo foram: Unidade de Apoio Cirúrgico (UAC), UTI cirúrgica/fígado, UTI do trauma, UTI da anestesiologia. As UTIs clínicas incluídas foram: UTI da pneumologia, UTI da clínica médica, UTI do Pronto Socorro, UTI da cirurgia plástica e UTI da divisão das moléstias infecciosas e parasitárias. Após serem avaliados pela equipe da nefrologia (avaliação solicitada espontaneamente pelo plantonista médico da UTI), os pacientes que tiveram 
indicação de começar a terapia de substituição renal e que obedeciam aos critérios de inclusão e exclusão poderiam ser incluídos no estudo.

3.2.1 Critérios de inclusão:

- Idade acima de 18 anos

- Pacientes que estavam internados nas UTIs que concordaram em participar do projeto.

3.2.2 Critérios de exclusão:

- Pacientes com idade inferior a 18 anos.

- Pacientes cuja indicação da hemodiálise não foi renal, tais como intoxicações exógenas, hipotermia ou hipertermia.

- Portadores de insuficiência renal crônica classe funcional V (pacientes que se encontravam em hemodiálise ambulatorial ou diagnosticada nesta internação com taxa de filtração glomerular menor ou igual a 15 $\mathrm{ml} / \mathrm{min}$ por $1,73 \mathrm{~m}^{2}$ )

- Transplantado renal

- Pacientes em cuidados paliativos 


\subsection{COLETA DE DADOS}

Durante o período de janeiro de 2012 a março de 2013 e janeiro de 2014 a setembro de 2014, foram coletados dados de exames clínicos e laboratoriais registrados em prontuários, de uma amostra não probabilística, por conveniência, dos pacientes internados em UTI do HCFMUSP, que iniciavam TRS após avaliação e indicação da equipe da nefrologia.

Os dados laboratoriais e de desfecho foram coletados do sistema de informática do Instituto Central do Hospital das Clínicas (ICHC), através do registro hospitalar dos pacientes.

Dados referentes à quantidade e tipos de hemodiálise realizada durante a internação hospitalar (quantas na UTI e quantas na enfermaria), permanência ou não em hemodiálise após a alta da UTI e hospitalar, foram coletados do formulário de atendimento do grupo dos agudos do serviço de nefrologia.

\subsection{VARIÁVEIS ANALISADAS}

Foram analisados dados referentes às características dos pacientes: idade, sexo, raça, peso, altura, doenças de base (diabetes, hipertensão, HIV, neoplasias malignas, neoplasias hematológicas, doença vascular periférica, aneurisma de aorta, DPOC, doença coronariana, ICC, transplantes prévios).

Também foram analisados dados referentes ao tipo de internação na UTI, clínica ou cirúrgica. Foi avaliado o motivo da admissão na UTI e procedência (centro cirúrgico, pronto socorro, UTI ou outros), data da internação hospitalar, data da internação na UTI e data do início de acompanhamento da equipe nefrologia. 
Vários fatores que poderiam contribuir para o episódio de IRA foram avaliados durante três dias que precederam a primeira hemodiálise: uso de antiinflamatórios não hormonais, diuréticos, inibidores da enzima de conversão da angiotensina, bloqueadores do receptor da angiotensina, contraste ou outras drogas nefrotóxicas como vancomicina, polimixina, tacrolimus ou outras. Foi avaliado também se o paciente recebeu transfusão sanguínea ou foi submetido a procedimento cirúrgico nos três dias que antecederam a primeira hemodiálise.

Os parâmetros clínicos e laboratoriais foram avaliados três dias antes do início da terapia dialítica, durante a primeira hemodiálise e nos seis dias seguintes. Os parâmetros clínicos avaliados incluíam presença ou não de edema, frequência cardíaca máxima e mínima, PAS máxima e mínima, PAM máxima e mínima, temperatura máxima e mínima, tipo de ventilação (espontânea, cateter nasal, máscara, VNI ou VM) e seus parâmetros relacionados (FiO2, PEEP). Foram anotados volume urinário de cada 6 horas e o balanço hídrico diário. Foi anotado o peso da admissão do paciente, caso não houvesse, anotado aquele informado pelo paciente ou seu familiar. No prontuário não constavam anotações do peso diário dos pacientes, já que não era rotina das UTIs. Foram registrados presença de sedação e uso de drogas vasoativas (tipo e dose). Quanto aos escores de gravidade, a escala de coma de Glasgow foi anotada e o SOFA (ANEXO A) e SAPS 3, "Simplified Acute Physiology Score 3" (ANEXO B) foram calculados. Dados bioquímicos como uréia, creatinina, hemograma, gasometria arterial, $\mathrm{Na}, \mathrm{K}$, cálcio total e ionizável, fósforo, CPK, albumina, PCR e lactato foram registrados. Dados de ureia e creatinina foram registrados por mais três dias além dos seis dias seguintes ao início da terapia dialítica. 
Foram anotados dados referentes à doença renal e a hemodiálise. Para avaliar presença de doença renal prévia foram anotados dados da $\mathrm{sCr}$ basal, $\mathrm{sCr}$ de referência (caso não tivesse informação da basal) e $\mathrm{sCr}$ da admissão hospitalar. Também foram anotadas as creatininas séricas da admissão UTI, da primeira diálise, da última diálise, da alta UTI, alta hospitalar ou óbito.

Dados referentes à terapia dialítica como indicação, modalidade, e dados da prescrição foram registrados. As indicações de diálise foram classificadas entre: sobrecarga hídrica, hipercalemia refratária ao tratamento clínico $(K>5,7)$, uremia (Uréia > $120 \mathrm{mg} / \mathrm{dL})$, acidose metabólica $(\mathrm{pH}<7,34$ e bicarbonato sérico $<18$ mEq/L na gasometria arterial) ou diálise de manutenção. Tipo da TRS se convencional, SLED ("Sustained Low-Efficiency Dialysis”) ou contínua; via de acesso para hemodiálise (cateter de duplo-lúmen temporário femoral, jugular ou subclávia); tipo de anticoagulação (heparina não fracionada, heparina de baixo peso, citrato de sódio, ou somente lavagem do sistema com soro fisiológico); tipo de capilar utilizado; concentrações dos eletrólitos do banho de diálise; tempo prescrito e tempo oferecido; parâmetros da hemodiálise como fluxo sangue, fluxo dialisato e ultrafiltração final; além do fluxo reposição e volume de lavagem se hemodiálise contínua.

Os dados foram anotados em relação à primeira hemodiálise e das hemodiálises dos seis dias seguintes. Com estes dados foram calculados as dose de diálise prescrita e oferecida dos primeiros sete dias de acordo com a modalidade da diálise como descrito abaixo: 
- Em métodos intermitentes (convencional ou SLED):

Com os dados da prescrição foi determinado, pela fórmula abaixo, o Kt/V prescrito e oferecido, sendo o volume de água corporal total determinado como uma fração do peso do paciente (para mulheres 0,55 e homens 0,60 ) e utilizando como depuração de uréia do dialisador aquele determinado pelo fabricante in vitro levando em consideração o fluxo sanguíneo de $200 \mathrm{ml} / \mathrm{min}$ ou $300 \mathrm{ml} / \mathrm{min}$.

Depuração de uréia do dialisador X tempo prescrito (minutos) $\mathrm{Kt} / \mathrm{V}$ prescrito $=$

Peso estimado $(\mathrm{kg}) \mathrm{X}(0,55$ ou 0,6$)$

Depuração de uréia do dialisador X tempo oferecido (minutos)

$\mathrm{Kt} / \mathrm{V}$ oferecido $=$

Peso estimado $(\mathrm{kg}) \mathrm{X}(0,55$ ou 0,6$)$

$\mathrm{O} \mathrm{Kt} / \mathrm{V}$ baseado nas equações de Daugirdas requer a coleta de uréia pré e pósdiálise, como se trata de um trabalho observacional a dose de diálise não foi avaliada por essas fórmulas. 
- Em métodos contínuos:

Nos métodos contínuos foi calculada a dose através do volume total de efluente utilizado durante as 24 horas de procedimento. $\mathrm{O}$ volume do efluente foi calculado pela soma do volume do dialisato mais o volume da solução de reposição.

Volume total de efluente prescrito em $24 \mathrm{~h}$

Dose prescrita $=$

Peso estimado $(\mathrm{kg}) / 24 \mathrm{~h}$

Volume total de efluente oferecido em $24 \mathrm{~h}$

Dose oferecida $=$

Peso estimado $(\mathrm{kg}) / 24 \mathrm{~h}$

Com relação aos desfechos clínicos hospitalares avaliados, incluíam dados de sobrevida, recuperação renal, duração da TRS e tempo de internação em UTI e hospitalar. Para isso foram anotados além das sCr já mencionadas, informações sobre alta da UTI em diálise, alta hospitalar em diálise ou óbito em diálise. Total de diálise convencional, SLED ou contínua em UTI e enfermaria.

\subsection{DESFECHOS}

O desfecho primário do estudo foi mortalidade hospitalar. Desfechos secundários incluem recuperação da função renal na alta hospitalar, tempo para 
controle metabólico e do balanço hídrico após início da terapia dialítica e dose de diálise durante o período de seguimento.

\subsection{ANÁLISE ESTATÍSTICA E CÁLCULO DA AMOSTRA}

O principal objetivo do estudo foi comparar a mortalidade hospitalar em relação ao momento de início da TRS de acordo com as diferentes definições de precocidade. O cálculo da amostra foi realizado com base no objetivo principal.

\subsubsection{Cálculo da amostra}

O tamanho da amostra para este estudo é baseado no desfecho mortalidade do objetivo principal. Para demonstrar a associação entre momento de início da terapia dialítica e mortalidade, o momento de início da diálise foi determinado como precoce ou tardio baseado em parâmetros clínicos e laboratoriais. Era esperado que a maioria dos pacientes (2:1) iniciasse a TRS com parâmetros considerados como tardio. O estudo compara dois grupos de pacientes (grupo de início precoce e início tardio). A hipótese nula é de que a mortalidade seria a mesma no grupo de início tardio e no grupo de início precoce. Com base na literatura e em dados da instituição estimou-se que a mortalidade dos pacientes submetidos à TRS seria de $60 \%$. Estimando que a mortalidade do grupo tardio fosse mais elevada, ao redor de $70 \%$, e do grupo precoce ao redor de 50\%, seria necessário 193 pacientes ( 77 pacientes no grupo precoce e 116 pacientes no grupo tardio) para mostrar uma diferença com poder de $80 \%$. Com esta amostra existe a probabilidade de $80 \%$ de o estudo mostrar um resultado estatisticamente significativo, permitindo concluir que a taxa de mortalidade é diferente entre pacientes com início precoce e tardio. O critério usado para significância estatística foi de 0,05 . 


\subsubsection{Análise estatística}

Os dados coletados foram inseridos em planilha eletrônica do Excel e a análise estatística realizada através do programa SPSS 20.0. As variáveis contínuas foram inicialmente avaliadas quanto a distribuição através da visualização de gráficos (histogramas e box-plots) e testes de normalidade (Shapiro-Wilk e Kolmogorov-Smirnoff). As variáveis distribuídas normalmente foram sumarizadas através da média e desvio padrão enquanto as distribuídas em forma não-normal foram sumarizadas através da mediana e distância interquartílica (P25\% a P75\%). As variáveis categóricas foram descritas utilizando-se frequências relativas. Para comparações de variáveis contínuas entre dois grupos, utilizamos os testes t de Student (distribuição normal) ou U de Mann-Whitney (distribuição não normal). As variáveis categóricas foram comparadas utilizando o Qui-Quadrado de Pearson ou Teste Exato de Fisher. As variáveis que atingiram significância estatística com p<0,2 na análise univariada, foram utilizadas no modelo de regressão logística. Regressão logística com seleção para trás (backward selection), usando $\mathrm{p}<0,20$, foi realizada para avaliação do impacto dos parâmetros selecionados na mortalidade dos pacientes. Foi adotado como nível para significância estatística um p-valor $<0,05$. 


\section{RESULTADOS}

Durante o período do estudo, foram analisados dados de 168 pacientes internados em UTI, que evoluíram com IRA e necessidade de hemodiálise.

\subsection{Características Clínicas}

As características gerais dos pacientes estão demonstradas na tabela 1. A mediana da idade dos pacientes foi 55 anos (43-65 anos). A comorbidade mais prevalente foi HAS, presente em 42,3\% dos pacientes estudados. A maioria dos pacientes foi internada em UTI por motivos clínicos $(58,3 \%)$.

Dos 94 pacientes que tinham $\mathrm{sCr}$ basal ou $\mathrm{sCr}$ referência com MDRD correspondente maior que $90 \mathrm{~mL} / \mathrm{min}$ por $1,73 \mathrm{~m}^{2}$, a mediana da $\mathrm{sCr}$ foi $0,87 \mathrm{mg} / \mathrm{dL}$ (0,71-1,00 mg/dL). Treze pacientes $(13,8 \%)$ tinham doença renal crônica prévia.

A presença de fatores associados ao desenvolvimento de IRA nos três dias que antecederam o início da terapia dialítica esta demonstrada na tabela 2. Noventa e nove pacientes $(58,9 \%)$ receberam diuréticos, 93 pacientes $(55,4 \%)$ receberam outras drogas nefrotóxicas como vancomicina e polimixina. Apenas 29 pacientes $(17,3 \%)$ receberam contraste e 10 pacientes $(6 \%)$ receberam IECA e/ou BRA. 
Tabela 1- Características gerais de toda população avaliada, dos pacientes que evoluíram para óbito e dos pacientes sobreviventes

\begin{tabular}{|c|c|c|c|c|}
\hline & $\begin{array}{c}\text { Total } \\
\mathbf{N}=168\end{array}$ & $\begin{array}{c}\text { Não } \\
\text { sobreviventes } \\
\mathbf{N}=\mathbf{1 2 3} \\
\end{array}$ & $\begin{array}{l}\text { Sobreviventes } \\
\quad \mathrm{N}=45\end{array}$ & $\mathbf{p}$ \\
\hline Sexo masculino & $107(63,7)$ & $78(63,4)$ & $29(64,4)$ & 0,526 \\
\hline Idade anos & $55(43-65)$ & $58(47-68)$ & $45(32,5-60)$ & $<0,001$ \\
\hline Raça branca & $94(56)$ & $72(58,5)$ & $22(48,9)$ & 0,280 \\
\hline \multicolumn{5}{|l|}{ Comorbidades } \\
\hline Hipertensão & $71(42,3)$ & $59(48)$ & $12(26,7)$ & 0,010 \\
\hline Diabete melito & $37(22)$ & $27(22)$ & $10(22,2)$ & 0,561 \\
\hline Doença renal crônica $^{\text {\& }}$ & $13(13,8)$ & $12(16,9)^{\alpha}$ & $1(4,3)^{\beta}$ & 0,130 \\
\hline Doença vascular periférica & $18(10,7)$ & $14(11,4)$ & $4(8,9)$ & 0,644 \\
\hline Doença hepática crônica & $33(19,6)$ & $25(20,3)$ & $8(17,8)$ & 0,449 \\
\hline Insuficiência cardíaca & $12(7,1)$ & $9(7,3)$ & $3(6,7)$ & 0,885 \\
\hline Câncer & $32(19)$ & $26(21,1)$ & $6(13,3)$ & 0,180 \\
\hline Transplante hepático & $15(8,9)$ & $9(7,3)$ & $6(13,3)$ & 0,180 \\
\hline $\mathrm{sCr}$ basal $(\mathrm{mg} / \mathrm{dL})^{\&}$ & $\begin{array}{c}0,87(0,71- \\
1,00)\end{array}$ & $\begin{array}{c}0,86(0,70- \\
1,04)\end{array}$ & $\begin{array}{c}0,89(0,74- \\
0,99)\end{array}$ & 0,912 \\
\hline MDRD basal $\left(\mathrm{ml} / \mathrm{min} / 1,73 \mathrm{~m}^{2}\right)^{\&}$ & $104(87-132)$ & $104(79-136)$ & $98(93-131)$ & 0,947 \\
\hline Tipo de Internação & & & & 0,178 \\
\hline Clínica & $98(58,3)$ & $67(54,5)$ & $31(68,9)$ & \\
\hline Cirúrgica & $39(23,2)$ & $32(26)$ & $7(15,6)$ & \\
\hline Cirúrgica de emergência & $31(18,5)$ & $24(19,5)$ & $7(15,6)$ & \\
\hline SOFA não renal & $9(4,5-13)$ & $11(9-13)$ & $9(6,5-11,5)$ & 0,017 \\
\hline SAPS 3 & $74(67-82)$ & $75(68-83)$ & $67(58-77)$ & 0,007 \\
\hline
\end{tabular}

NOTA: Variáveis categóricas expressas em número absoluto e percentagem e variáveis contínuas expressas como mediana e intervalo interquartil (P25-P75); MDRD,

"Modification of Diet in Renal Disease Study"; N, número; $\mathrm{sCr}$, creatinina sérica; \& Valores referentes a 94 pacientes; ${ }^{\alpha}$ Valores referentes a 71 pacientes não-sobreviventes; ${ }^{\beta}$ Valores referentes a 23 pacientes sobreviventes 
Tabela 2 - Fatores associados ao desenvolvimento de IRA nos três dias anteriores ao início da terapia dialítica

\begin{tabular}{|c|c|c|c|c|}
\hline & $\begin{array}{c}\text { Total } \\
\mathrm{N}=168\end{array}$ & $\begin{array}{c}\text { Não sobreviventes } \\
\mathbf{N}=123\end{array}$ & $\begin{array}{c}\text { Sobreviventes } \\
\mathrm{N}=45\end{array}$ & $\mathbf{p}$ \\
\hline Hipoalbuminemia $^{(1)}$ & $145(92,4)^{(2)}$ & $108(95,6)$ & $37(84,1)$ & 0,022 \\
\hline Diurético & $99(58,9)$ & $73(59,3)$ & $26(57,8)$ & 0,496 \\
\hline IECA/BRA & $10(6)$ & $4(3,3)$ & $6(13,3)$ & 0,014 \\
\hline Contraste & $29(17,3)$ & $16(13)$ & $13(28,9)$ & 0,017 \\
\hline Transfusão sanguínea & $83(49,4)$ & $59(48)$ & $24(53,3)$ & 0,329 \\
\hline Drogas nefrotóxicas & $93(55,4)$ & $75(61)$ & $18(40)$ & 0,012 \\
\hline PAM mínima & $62(57-67)$ & $61(56-66)$ & $64(57-70)$ & 0,071 \\
\hline
\end{tabular}

NOTA: IECA, inibidor da enzima conversora da angiotensina; BRA, bloqueador do receptor da angiotensina II; IRA, injúria renal aguda; PAM, pressão arterial média; Variáveis categóricas expressas em número absoluto e percentagem, variáveis contínuas expressas como mediana e intervalo interquartil (P25-P75);

(1) Albumina sérica $<3,5 \mathrm{mg} / \mathrm{dL}$ durante internação hospitalar

(2) de um total 157; onze pacientes sem essa informação

\subsection{Parâmetros clínicos e laboratoriais no momento do início da TRS}

No início da TRS, dos 168 pacientes analisados, 138 (82,1\%) estavam em ventilação mecânica e $134(79,8 \%)$ recebiam drogas vasoativas. Em relação à sedação, 54 pacientes $(32,1 \%)$ não estavam sedados no início da TRS. As medianas dos escores de gravidade no momento do início da diálise foram 74 (67-82) e 14 (1117) para SAPS 3 e SOFA, respectivamente, como demonstrado na tabela 3. Com relação às características laboratoriais, os pacientes começaram a terapia dialítica com mediana da $\mathrm{sCr}$ de 3,81 mg/dL (2,59-5,11 mg/dL) e mediana da uréia de 138 $\mathrm{mg} / \mathrm{dL}$ (98-182 mg/dL). A maioria dos pacientes $(58,7 \%)$ apresentava edema, seja localizado ou generalizado, no início da TRS. A mediana da diurese em 24 horas foi 
$450 \mathrm{ml}$ (195-1000 ml) e a mediana do balanço hídrico acumulado no início da TRS foi $2862 \mathrm{ml}(1088-5053 \mathrm{ml})$.

No momento do início da TRS, utilizando o critério da sCr, 115 pacientes $(68,8 \%)$ foram classificados como AKIN 3. Pelo critério do débito urinário 85 pacientes $(51,2 \%)$ foram classificados como AKIN 3. Utilizando-se ambos os critérios, 141 pacientes $(83,9 \%)$ estavam em AKIN 3. 
Tabela 3 - Características clínicas e laboratoriais no início da terapia renal substitutiva

\begin{tabular}{|c|c|c|c|c|}
\hline Variáveis & $\begin{array}{c}\text { Total } \\
\mathrm{N}=168\end{array}$ & $\begin{array}{c}\text { Não } \\
\text { sobreviventes } \\
\mathbf{N}=\mathbf{1 2 3}\end{array}$ & $\begin{array}{l}\text { Sobreviventes } \\
\quad \mathrm{N}=\mathbf{4 5}\end{array}$ & $\mathbf{p}$ \\
\hline Ventilação mecânica & $138(82,1)$ & $111(90,2)$ & $27(60)$ & $<0,001$ \\
\hline Drogas vasoativas & $134(79,8)$ & $107(87)$ & $27(60)$ & $<0,001$ \\
\hline Ausência de sedação & $54(32,1)$ & $32(26)$ & $22(48,9)$ & 0,005 \\
\hline Edema $^{(1)}$ & & & & 0,581 \\
\hline Localizado & $58(47,9)$ & $42(48,8)$ & $16(45,7)$ & \\
\hline Anasarca & $13(10,8)$ & $11(12,8)$ & $2(5,7)$ & \\
\hline SAPS 3 escore & $74(67-82)$ & $75(68-83)$ & $67(58-77)$ & 0,007 \\
\hline SOFA escore & $14(11-17)$ & $14(11-17)$ & $12(10-15)$ & 0,044 \\
\hline PAM mínima (mmHg) & $67(61-73)$ & $61(56-66)$ & $64(58-70)$ & 0,071 \\
\hline PEEP & $8(5-10)$ & $8(6-10)$ & $6(0-10)$ & 0,016 \\
\hline $\mathrm{PaO} 2 / \mathrm{FiO} 2$ & $261(164-367)$ & $257(161-336)$ & $284(182-387)$ & 0,359 \\
\hline Diurese $24 \mathrm{~h}(\mathrm{ml})$ & $450(195-1000)$ & $420(165-900)$ & $600(350-1200)$ & 0,110 \\
\hline Balanço hídrico diário (ml) & $809(-267-2216)$ & $1064(-53-2273)$ & $270(-820-1301)$ & 0,044 \\
\hline BH acumulado até início da TRS & $2862(1088-5053)$ & $3090(1580-5395)$ & $1632(84-3910)$ & 0,004 \\
\hline Uréia (mg/dL) & $138(98-182)$ & $134(100-181)$ & $150(89-184)$ & 0,957 \\
\hline Creatinina $(\mathrm{mg} / \mathrm{dL})$ & $3,81(2,59-5,11)$ & $3,69(2,61-4,69)$ & $4,03(2,54-5,28)$ & 0,496 \\
\hline PCR (mg/dL) & $168(75-292)$ & $143(73-278)$ & $215(98-316)$ & 0,164 \\
\hline $\mathrm{pH}$ arterial & $7,33(7,27-7,38)$ & $7,33(7,26-7,38)$ & $7,33(7,29-7,39)$ & 0,458 \\
\hline Bicarbonato arterial (mEq/L) & $19(16-22)$ & $19(15-21)$ & $20(16-23)$ & 0,930 \\
\hline Lactato $(\mathrm{mg} / \mathrm{dL})$ & $31(22-44)$ & $32(25-46)$ & $27(21-39)$ & 0,158 \\
\hline AKIN pela $\mathrm{sCr}$ & & & & 0,119 \\
\hline 1 & $33(19,8)$ & $26(78,8)$ & $7(21,2)$ & \\
\hline 2 & $19(11,4)$ & $17(89,5)$ & $2(10,5)$ & \\
\hline 3 & $115(68,8)$ & $79(68,7)$ & $36(31,3)$ & \\
\hline AKIN pelo débito urinário & & & & 0,375 \\
\hline 1 & $46(27,7)$ & $34(74)$ & $12(26)$ & \\
\hline 2 & $35(21,1)$ & $21(60)$ & $14(40)$ & \\
\hline 3 & $85(51,2)$ & $67(78,8)$ & $18(21,2)$ & \\
\hline AKIN pela sCr ou débito urinário & & & & 0,944 \\
\hline 1 & $14(8,4)$ & $10(71,4)$ & $4(28,6)$ & \\
\hline 2 & $13(7,7)$ & $10(76,9)$ & $3(23,1)$ & \\
\hline 3 & $141(83,9)$ & $103(73)$ & $38(27)$ & \\
\hline
\end{tabular}

NOTA: N, número; PAM, pressão arterial média; $\mathrm{PCR}$, proteína $\mathrm{C}$ reativa; $\mathrm{PEEP}$, pressão positiva expiratória final; SAPS, Simplified Acute Physiology Score; SOFA, Sequential Organ Failure Assessment; Variáveis categóricas expressas em número absoluto e percentagem e variáveis contínuas expressas como mediana e intervalo interquartil (P25P75); ${ }^{(1)}$ de um total de 121; 47 pacientes sem essa informação 


\subsection{Características da TRS}

Com relação às características da TRS, os métodos contínuos foram a modalidade de diálise mais frequentemente realizada, $62,5 \%$, seguido dos métodos híbridos (SLED) em 29,8\%, e apenas 7,7\% realizaram hemodiálise convencional (tabela 4). A indicação de diálise mais frequente foi uremia (36,3\%), seguida de sobrecarga hídrica $(35,1 \%)$. O flush de soro fisiológico no sistema foi o método de anticoagulação em $46,4 \%$ dos casos; $44,6 \%$ utilizaram citrato e apenas $8,9 \%$ heparina. As vias de acesso mais frequentemente utilizadas para TRS foram veia femoral direita $(36,9 \%)$ e veia jugular direita $(24,4 \%)$. Na primeira hemodiálise a mediana do Kt/V oferecido nas hemodiálises intermitentes foi 1,49 $(1,06-1,93)$ e a mediana da dose oferecida nos métodos contínuos foi $34,8 \mathrm{ml} / \mathrm{kg} / \mathrm{h}$ (31,1-44,2 $\mathrm{ml} / \mathrm{kg} / \mathrm{h})$.

Nas hemodiálises subsequentes a mediana da dose oferecida nos métodos contínuos foi maior que $28 \mathrm{ml} / \mathrm{kg} / \mathrm{h}$ e o $\mathrm{Kt} / \mathrm{V}$ oferecido maior que 1,43 nos métodos intermitentes. Após a primeira diálise, sobrecarga hídrica era o motivo de indicação de hemodiálise mais frequente até o quarto dia quando a diálise de manutenção superou a indicação de diálise por sobrecarga hídrica. Os métodos contínuos permaneceram sendo os mais frequentemente utilizados nos dias de acompanhamento somente sendo superado pelos métodos intermitentes no quinto dia de seguimento. 
Tabela 4 - Características da primeira terapia renal substitutiva

\begin{tabular}{|c|c|c|c|c|}
\hline Variáveis & $\begin{array}{l}\text { Total } \\
\mathbf{N}=168\end{array}$ & $\begin{array}{c}\text { Não sobreviventes } \\
\mathbf{N}=123\end{array}$ & $\begin{array}{c}\text { Sobreviventes } \\
\quad \mathbf{N}=45\end{array}$ & $\mathbf{p}$ \\
\hline Modalidade contínua & $105(62,5)$ & $80(65)$ & $25(55,5)$ & 0,172 \\
\hline Modalidade Intermitente & & & & 0,172 \\
\hline Convencional & $13(7,7)$ & $8(6,5)$ & $5(11,1)$ & \\
\hline SLED & $50(29,8)$ & $35(28,5)$ & $15(33,3)$ & \\
\hline Dose oferecida $(\mathrm{ml} / \mathrm{kg} / \mathrm{h})$ & $34,8(31,1-44,2)$ & $35,1(30,6-42,6)$ & $35,7(24,6-40,4)$ & 0,114 \\
\hline $\mathrm{Kt} / \mathrm{V}$ prescrito & $1,61(1,18-2,10)$ & $1,68(1,37-2,1)$ & $1,54(1,22-1,84)$ & 0,024 \\
\hline $\mathrm{Kt} / \mathbf{V}$ oferecido & $1,49(1,06-1,93)$ & $1,48(1,29-1,93)$ & $1,50(1,17-1,81)$ & 0,457 \\
\hline Indicação & & & & 0,524 \\
\hline Sobrecarga hídrica & $59(35,1)$ & $44(35,8)$ & $15(33,3)$ & \\
\hline Hipercalemia $^{*}$ & $13(7,7)$ & $8(6,5)$ & $5(11,1)$ & \\
\hline Uremia $^{\#}$ & $61(36,3)$ & $41(33,3)$ & $20(44,4)$ & \\
\hline Acidose ${ }^{\&}$ & $35(20,8)$ & $30(24,4)$ & $5(11,1)$ & \\
\hline \multicolumn{5}{|l|}{ UF } \\
\hline Contínua (ml) & $206(67-534)$ & 1025 (249-1673) & 1758 (1316-2199) & $<0,001$ \\
\hline Intermitente (ml) & $1000(625-2000)$ & $1500(1000-2000)$ & $1750(1255-2250$ & 0,176 \\
\hline Anticoagulação & & & & 0,805 \\
\hline Heparina & $15(8,9)$ & $12(9,8)$ & $3(6,7)$ & \\
\hline Citrato & $75(44,6)$ & $55(45,5)$ & $20(44,4)$ & \\
\hline Flush SF & $78(46,4)$ & $56(45,5)$ & $22(48,9)$ & \\
\hline Via de acesso & & & & 0,005 \\
\hline VJD & $41(24,4)$ & $23(18,7)$ & $18(40)$ & \\
\hline VJE & $38(22,6)$ & $24(19,5)$ & $14(31,1)$ & \\
\hline VFD & $62(36,9)$ & $54(43,9)$ & $8(17,8)$ & \\
\hline VFE & $22(13,1)$ & $19(15,4)$ & $3(6,7)$ & \\
\hline VSD & $3(1,8)$ & $2(1,6)$ & $1(2,2)$ & \\
\hline VSE & $2(1,2)$ & $1(0,8)$ & $1(2,2)$ & \\
\hline
\end{tabular}

NOTA: N, número; SF, soro fisiológico; UF, ultrafiltração; VJD, veia jugular direita; VJE, veia jugular esquerda; VSD, veia subclávia direita; VSE, veia subclávia esquerda; VFD, veia femoral direita; VFE, veia femoral esquerda. ${ }^{*} \mathrm{~K}^{+}>5,7 \mathrm{mEq} / \mathrm{L}^{*}$ Uréia $>120 \mathrm{mEq} / \mathrm{L}^{\text {\& }}$ Bicarbonato $<18 \mathrm{mEq} / \mathrm{L} \mathrm{e} \mathrm{pH}<7,34$ 


\subsection{Evolução de parâmetros clínicos e laboratoriais antes e após início da TRS}

Parâmetro clínico como o critério de gravidade SOFA não renal não variou muito desde o início do acompanhamento (três dias antes do início da TRS) até os sete dias subsequentes como ilustrado na figura 2. No dia do início da TRS 82,1\% dos pacientes estavam em ventilação mecânica e 79,8\% em uso de droga vasoativa. No sexto dia após este início 58,3\% dos pacientes estavam em VM e 46,1\% necessitavam droga vasoativa (figura 3). Não houve diferença na relação $\mathrm{PaO} 2 / \mathrm{FiO} 2$ e no lactato sérico nos dias de seguimento. O balanço hídrico acumulado foi progressivamente menor após o início da terapia dialítica. 


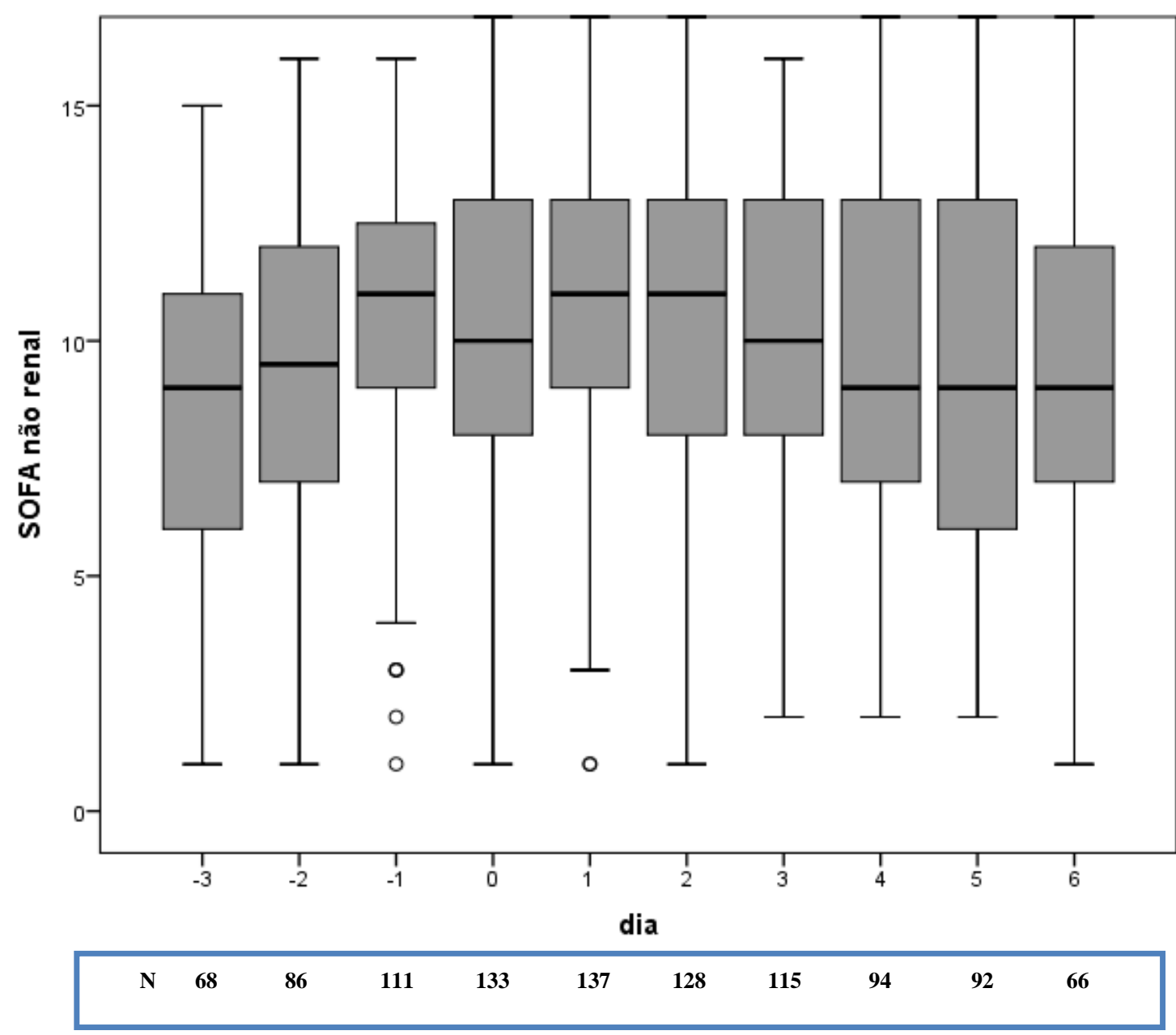

Figura 2- Evolução do escore SOFA não renal no período de seguimento dos pacientes em terapia renal substitutiva 


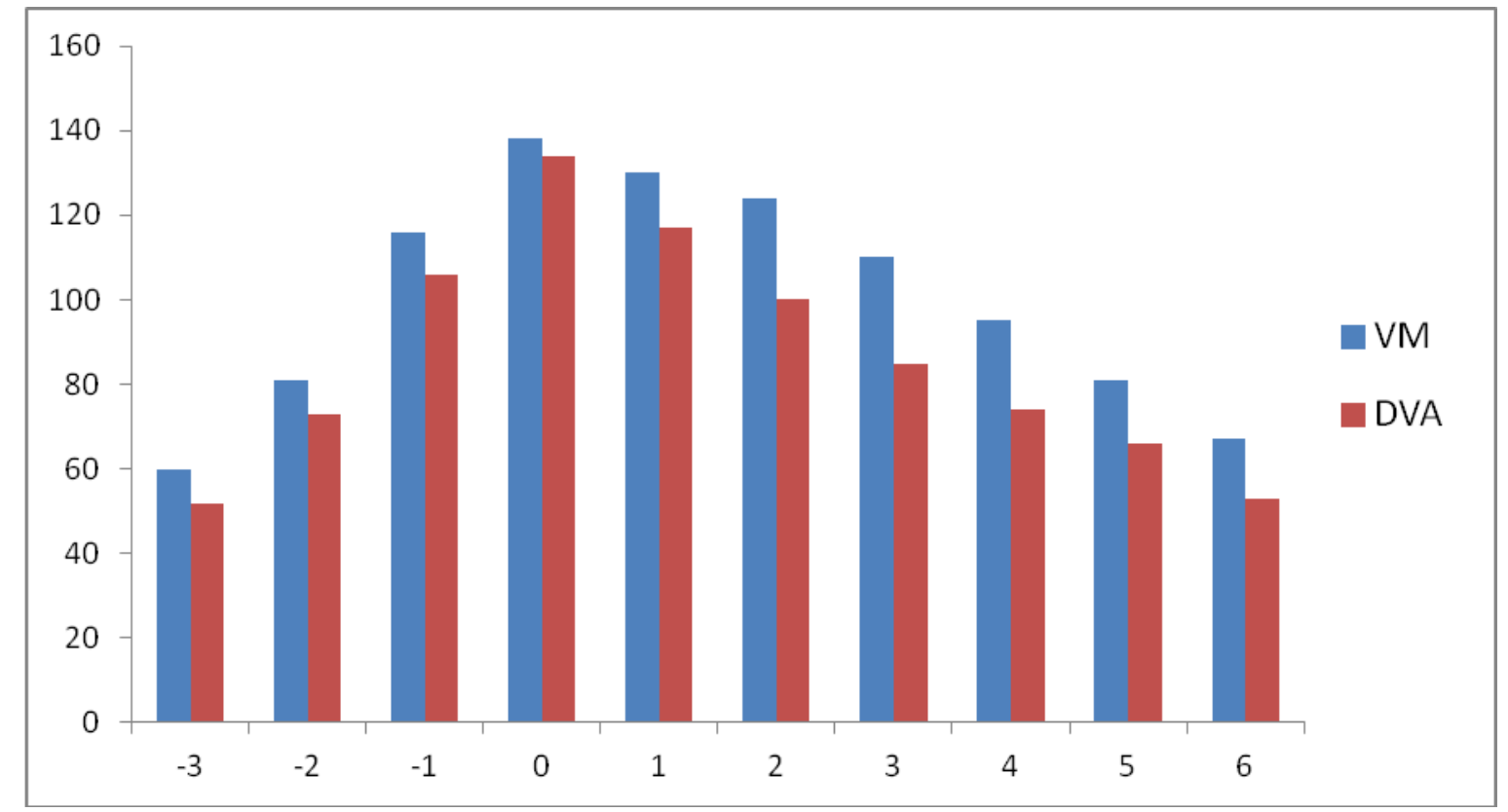

$\begin{array}{llllllllllll}\text { VM } & \text { N } & 133 & 149 & 162 & 168 & 161 & 155 & 146 & 134 & 126 & 115 \\ \text { DVA } & \text { N } & 132 & 149 & 162 & 168 & 161 & 155 & 146 & 134 & 126 & 115\end{array}$

Figura 3 - Número de pacientes em uso de ventilação mecânica e droga vasoativa no período de acompanhamento

NOTA: DVA, droga vasoativa; N, número total de pacientes; VM, ventilação mecânica

\subsection{Controle metabólico e do balanço hídrico após início da TRS}

A mediana do tempo para atingir níveis de uréia menores que $100 \mathrm{mg} / \mathrm{dL}$ foi de 2 dias (1-4 dias). A mediana do tempo para atingir controle acidobásico foi de 2 dias (1-3 dias). O controle do balanço hídrico foi alcançado no primeiro dia de tratamento (1-2 dias). 
4.6 Características clínicas, laboratoriais e da TRS em sobreviventes e não sobreviventes

A mortalidade hospitalar dos pacientes submetidos à TRS foi $73,2 \%$. Na figura 4 é apresentado um fluxograma dos desfechos alta hospitalar e óbito, e as taxas de recuperação da função renal dos sobreviventes.

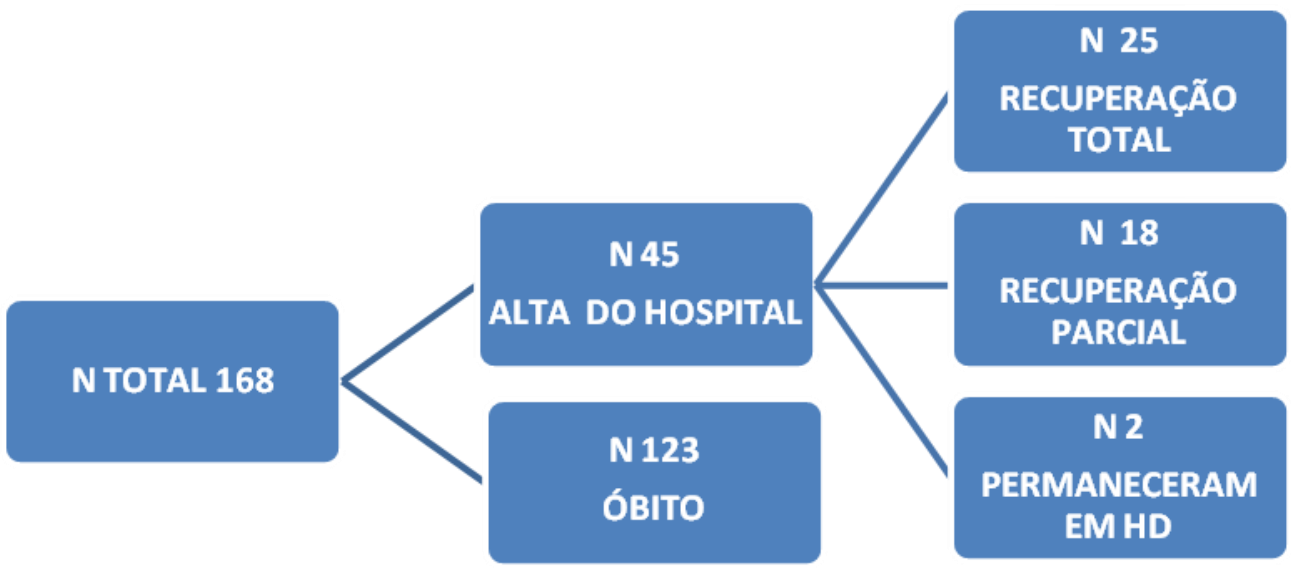

Figura 4 - Fluxograma da recuperação função renal e mortalidade na alta hospitalar 
A mediana da idade dos pacientes foi maior no grupo que evoluiu para óbito quando comparado com os sobreviventes (58 anos vs.45 anos, $\mathrm{p}<0,001)$. Quarenta e oito por cento dos pacientes que evoluíram para óbito eram hipertensos comparados com $26,7 \%$ dos pacientes sobreviventes $(\mathrm{p}=0,010)$.

Os pacientes que não sobreviveram tinham mediana do escore de gravidade SAPS 3 e SOFA não-renal maior que os pacientes sobreviventes, com significância estatística ( 75 vs. $67, \mathrm{p}=0,007$ e 11 vs. $9, \mathrm{p}=0,017$ respectivamente).

Setenta e cinco pacientes (61\%) que evoluíram para óbito receberam drogas nefrotóxicas nos três dias que precederam o início da TRS, enquanto 18 pacientes (40\%) sobreviventes receberam essas medicações $(\mathrm{p}=0,012)$.

Aproximadamente $29 \%$ dos sobreviventes receberam contraste, comparados com $13 \%$ dos não sobreviventes $(\mathrm{p}=0,017)$. Apenas seis pacientes $(13,3 \%)$ sobreviventes e quatro pacientes $(3,3 \%)$ que evoluíram para óbito receberam IECA e/ou BRA, porém com significância estatística $(\mathrm{p}=0,014)$.

A maioria dos pacientes que estavam em ventilação mecânica no início da terapia dialítica evoluiu para óbito. Como demonstrado na tabela 3, 90,2\% dos pacientes não sobreviventes comparados com $60 \%$ dos sobreviventes estavam em ventilação mecânica no início da TRS $(\mathrm{p}<0,001)$. Resultado semelhante em relação à presença de drogas vasoativas no início da TRS. Oitenta e sete por cento dos pacientes não sobreviventes recebiam drogas vasoativas comparados com $60 \%$ dos sobreviventes, também com significância estatística $(\mathrm{p}<0,001)$. Com relação à ausência de sedação, $26 \%$ dos pacientes que evoluíram para óbito e 48,9\% dos pacientes sobreviventes, estavam sem sedação no início da TRS $(p=0,005)$. 
A mediana do balanço hídrico diário no início da TRS foi maior, com significância estatística, nos pacientes que evoluíram para óbito quando comparados como os pacientes sobreviventes (1064 $\mathrm{ml} v s .270 \mathrm{ml}, \mathrm{p}=0,044)$. Quando avaliamos o BH acumulado dos 3 dias antes até o início da TRS, nos pacientes que evoluíram para óbito, o acúmulo de fluidos também foi significativamente maior comparado aos sobreviventes (3090 $\mathrm{ml}$ vs. $1632 \mathrm{ml}, \mathrm{p}=0,004)$.

A modalidade contínua foi mais frequente no grupo que evoluiu para óbito do que no grupo dos sobreviventes, mas sem significância estatística (65\% vs. 55,5\%, $\mathrm{p}=0,172$ ). Os pacientes que foram selecionados para diálise contínua tinham SOFA não renal e SAPS 3 maior que os pacientes selecionados para diálise intermitente, com $\mathrm{p}=0,018$ e $\mathrm{p}=0,043$ respectivamente. A indicação de TRS mais frequente no grupo de pacientes não-sobreviventes foi sobrecarga hídrica $(35,8 \%)$ e uremia $(44,4 \%)$ no grupo dos pacientes sobreviventes $(\mathrm{p}=0,524)$.

Analisando dose de diálise e mortalidade hospitalar, os pacientes não sobreviventes tiveram mediana de dose de diálise e $\mathrm{Kt} / \mathrm{V}$ discretamente menores quando comparados aos sobreviventes $(35,1 \mathrm{ml} / \mathrm{kg} / \mathrm{h}$ vs. $35,7 \mathrm{ml} / \mathrm{kg} / \mathrm{h}$ e 1,48 vs. 1,50 respectivamente).

Em relação ao controle metabólico os sobreviventes apresentaram maior tempo para atingir o controle de ureia, 3 dias vs. 2 dias comparado com os não sobreviventes. Não houve diferença em relação ao tempo para controle do bicarbonato sérico entre sobreviventes e não sobreviventes, enquanto os não sobreviventes apresentaram maior tempo para controle dos níveis de $\mathrm{pH}$ (3 dias $v s .2$ dias). Não houve diferença no tempo para atingir o controle do $\mathrm{BH}$ entre sobreviventes e não sobreviventes ( 1 dia vs. 1 dia, $\mathrm{p}=0,750)$. 
A mediana de UF, quando todas as diálises foram analisadas, foi a mesma entre os sobreviventes e não sobreviventes $(\mathrm{p}=0,515)$. Porém quando apenas as diálises contínuas foram analisadas, a mediana de UF entre os sobreviventes foi maior (1758 ml) que a dos não sobreviventes (1025 ml), p<0,001 (figura 5). Não houve diferença na mediana da UF dos métodos intermitentes em relação à mortalidade. A figura 5 mostra ainda que, os pacientes que fizeram diálise intermitente raramente atingiram UF > $2000 \mathrm{ml}$ nos primeiros dias, independente da gravidade e independente do desfecho óbito. 


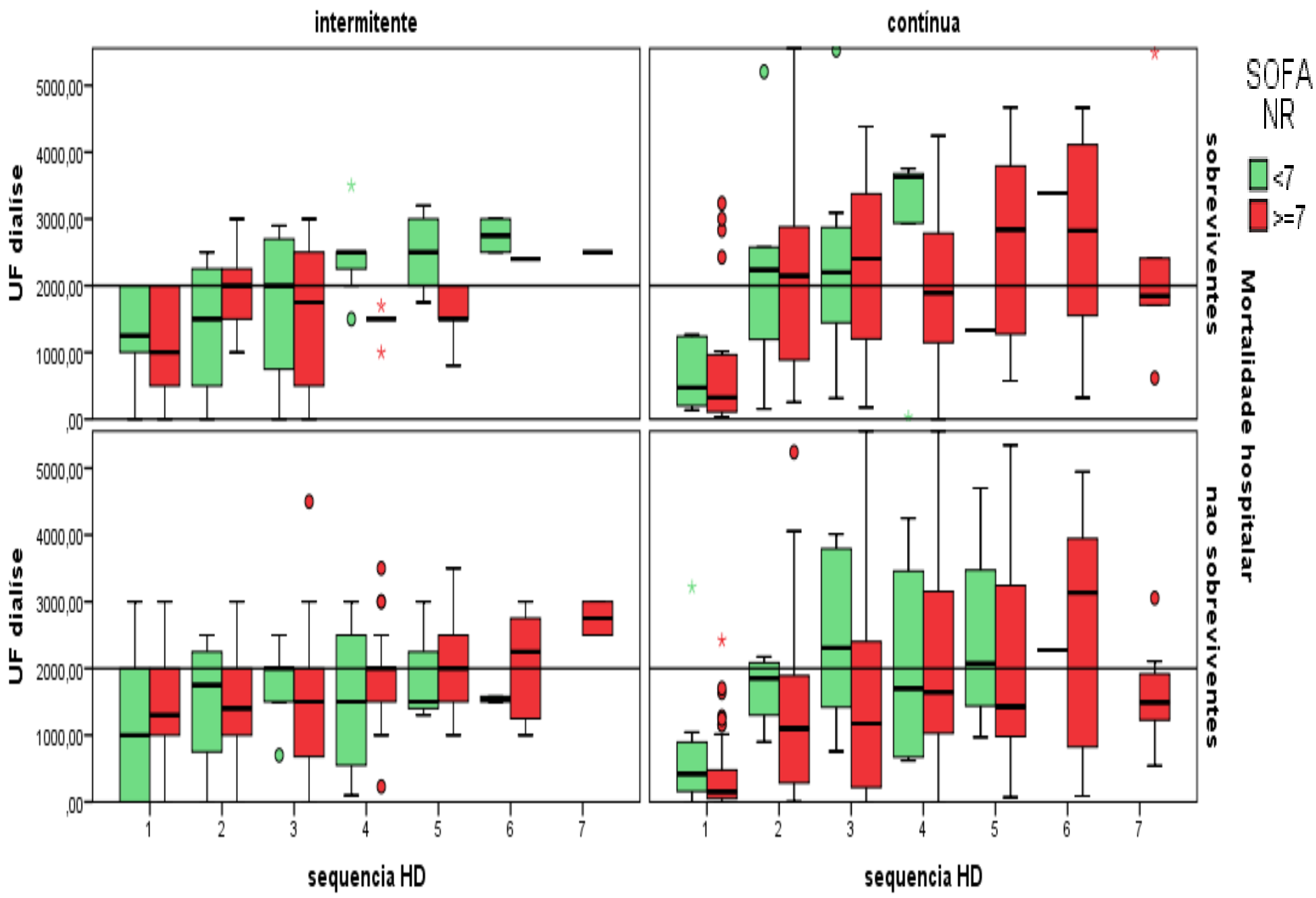

Figura 5 - Mediana da UF nos dias subsequentes à primeira diálise em relação à mortalidade, modalidade de diálise e score de gravidade SOFA não renal 
A Figura 6 ilustra a variação do $\mathrm{BH}$ acumulado entre os pacientes sobreviventes e não sobreviventes, no período de seguimento. Os pacientes que foram selecionados para diálise intermitente, tinham $\mathrm{BH}$ menor comparados com os que foram selecionados para diálise contínua.

A porcentagem de pacientes não sobreviventes foi maior quanto maior a porcentagem de BH em relação ao peso como demonstrado na figura 7.

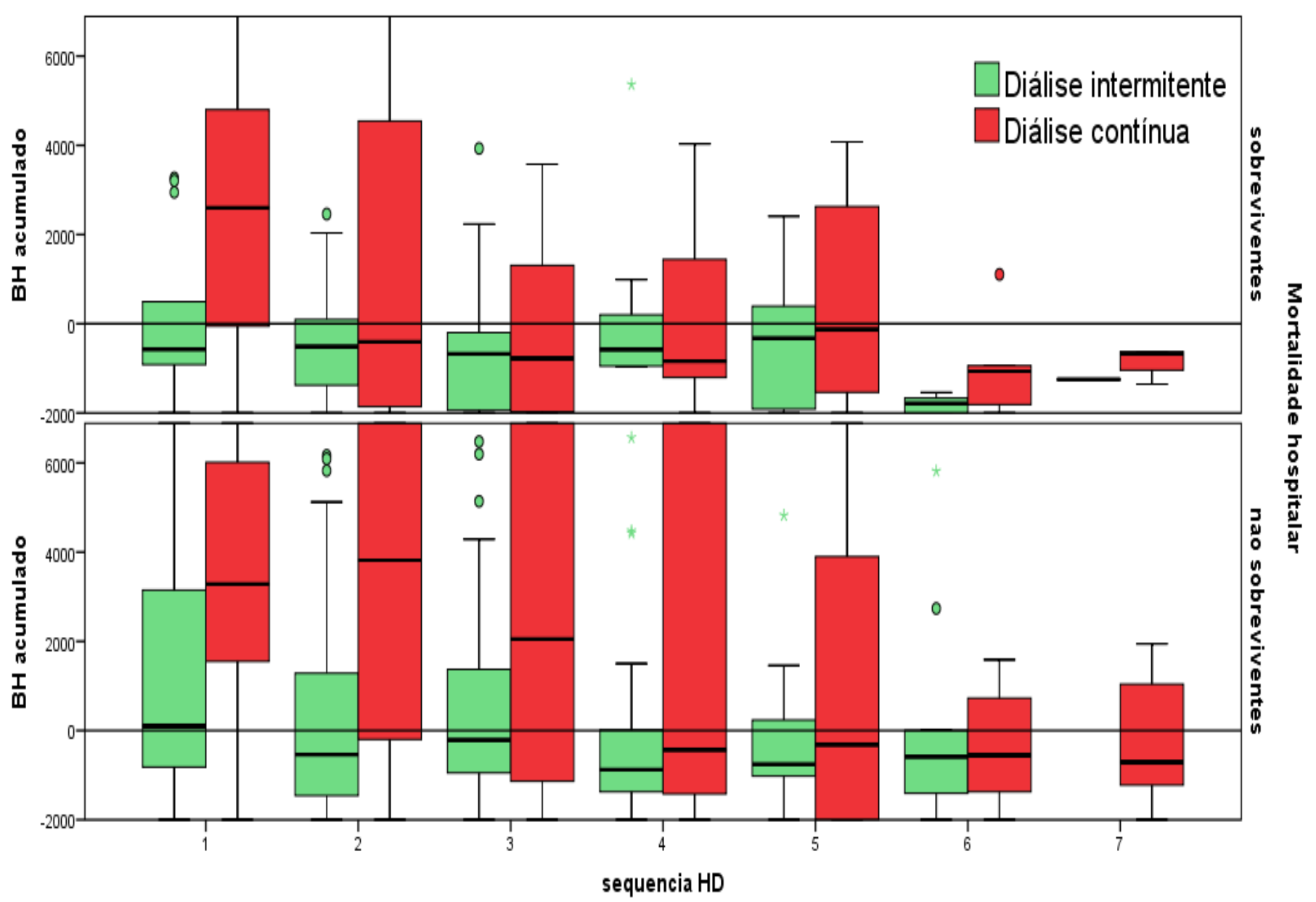

Figura 6 - Variação do balanço hídrico acumulado em relação à mortalidade e método dialítico no período de seguimento dos pacientes 


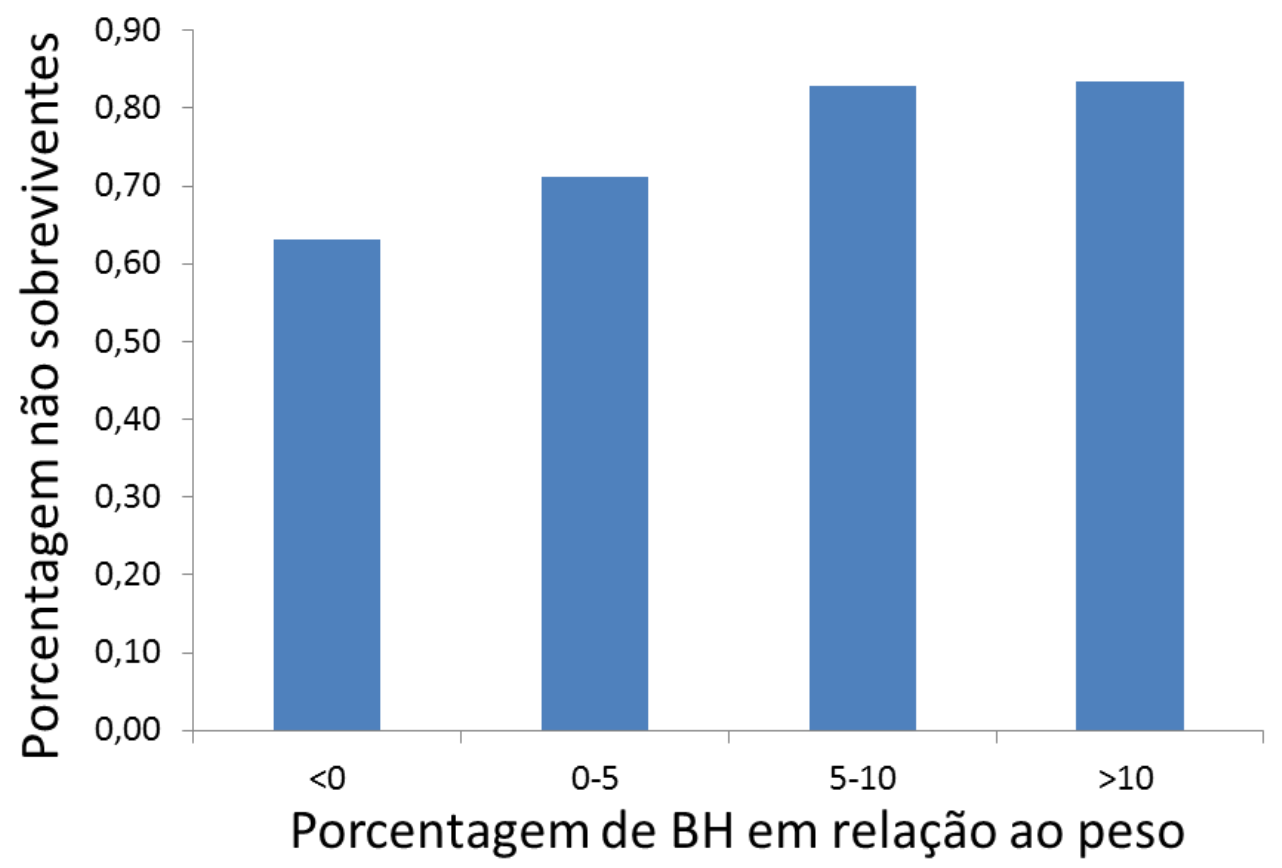

Figura 7 - Porcentagem de $\mathrm{BH}$ em relação ao peso e mortalidade

\subsection{Parâmetros para início da TRS e mortalidade}

Quando a uréia maior que $120 \mathrm{mg} / \mathrm{dL}$ no início da TRS foi utilizado para avaliar resultados, não houve diferença na mortalidade hospitalar entre os que começaram com uréia maior que $120 \mathrm{mg} / \mathrm{dL}$ e os que começaram com ureia menor que este valor. Foram avaliados outros níveis de ureia como ponto de corte para início da TRS, mas também não mostrou diferença. A curva ROC da uréia para predizer mortalidade foi 0,5 .

Dos 123 pacientes que foram a óbito, 103 pacientes $(83,7 \%)$ iniciaram TRS com AKIN 3. Não houve diferença em relação à mortalidade hospitalar entre os 
pacientes que iniciaram a terapia dialítica com AKIN 3 quando comparados com os pacientes que iniciaram com AKIN 1 ou $2(\mathrm{p}=0,560)$.

O menor tempo de internação em UTI no início da TRS esteve associado com menor mortalidade hospitalar. Pacientes que iniciaram a TRS antes de 2 dias da internação em UTI tiveram menor mortalidade hospitalar, $62,7 \%$ vs. $73,9 \%$ quando a TRS foi iniciada entre 2 dias e 5 dias, vs. 82,5\% quando TRS iniciada após 5 dias de admissão em UTI, $\mathrm{p}=0,046$, como ilustrado na figura 8 .

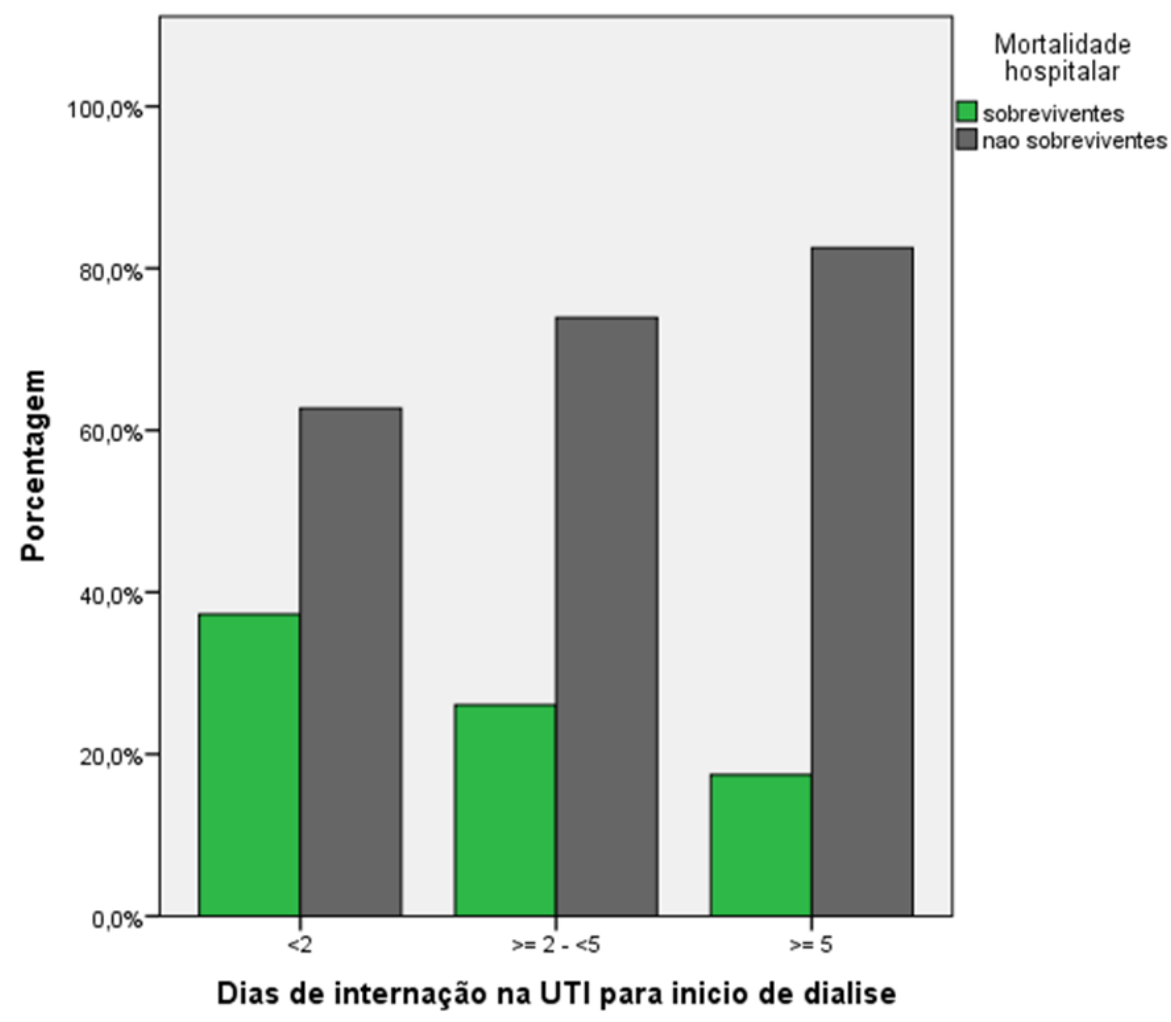

Figura 8 - Mortalidade hospitalar em relação ao tempo de UTI no momento de início da terapia renal 
Foi realizada análise multivariada, incluindo nove potenciais co-variáveis de confusão que foram associados com mortalidade na análise univariada: idade, tipo de internação, presença de hipoalbuminemia, HAS, câncer, DRC, sedação, droga vasoativa e tempo de internação na UTI no início da TRS. As variáveis, idade, sedação e tempo de internação na UTI no início da TRS estiveram associados de forma independente à mortalidade hospitalar (tabela 5).

Tabela 5 - Análise multivariada para mortalidade hospitalar

\begin{tabular}{lccc}
\hline \multicolumn{1}{c}{ Variáveis } & OR & $\mathbf{9 5 \%}$ IC & $\mathbf{p}$ \\
\hline Idade & 1,05 & $1,0-1,1$ & $<0,001$ \\
$\begin{array}{l}\text { Ausência de sedação } \\
\text { Tempo de internação em }\end{array}$ & 0,10 & $0,02-0,50$ & 0,005 \\
UTI até início da TRS & & & 0,046 \\
$\quad \mathbf{2}$ dias & 1,00 & & \\
$\mathbf{2} \mathbf{2}<\mathbf{5}$ dias & 4,70 & $1,0-21,9$ & \\
$\mathbf{2} \mathbf{5}$ dias & 20,93 & $3,1-139,3$ & \\
\hline
\end{tabular}

NOTA: IC, intervalo de confiança; OR, odds ratio;

\subsection{Recuperação da função renal em relação às características clinicas,} laboratoriais e da TRS

Dos 45 pacientes que receberam alta do hospital $25(55,5 \%)$ apresentavam recuperação total da função renal. Dezoito pacientes (40\%) recuperaram parcialmente a função renal e apenas dois pacientes (4,5\%) permaneceram em tratamento dialítico após a alta hospitalar.

Em relação à recuperação da função renal, foram analisadas todas as variáveis descritas na tabela 3 e não houve diferença estatística quando comparados os 
pacientes que recuperaram totalmente a função renal com os pacientes que recuperaram parcialmente.

A mediana da dose de diálise nos pacientes que recuperaram a função renal total foi maior em métodos contínuos e menor em diálise intermitente quando comparados com os pacientes que recuperaram parcialmente a função renal na alta hospitalar $(36,6 \mathrm{ml} / \mathrm{kg} / \mathrm{h}$ vs. $26,4 \mathrm{ml} / \mathrm{kg} / \mathrm{h}, \mathrm{p}=0,208$ e 1,45 vs. $1,65, \mathrm{p}=0,592) . \mathrm{A}$ figura 9 ilustra a mediana das doses de diálise oferecidas dos métodos contínuos, com relação à recuperação da função renal.

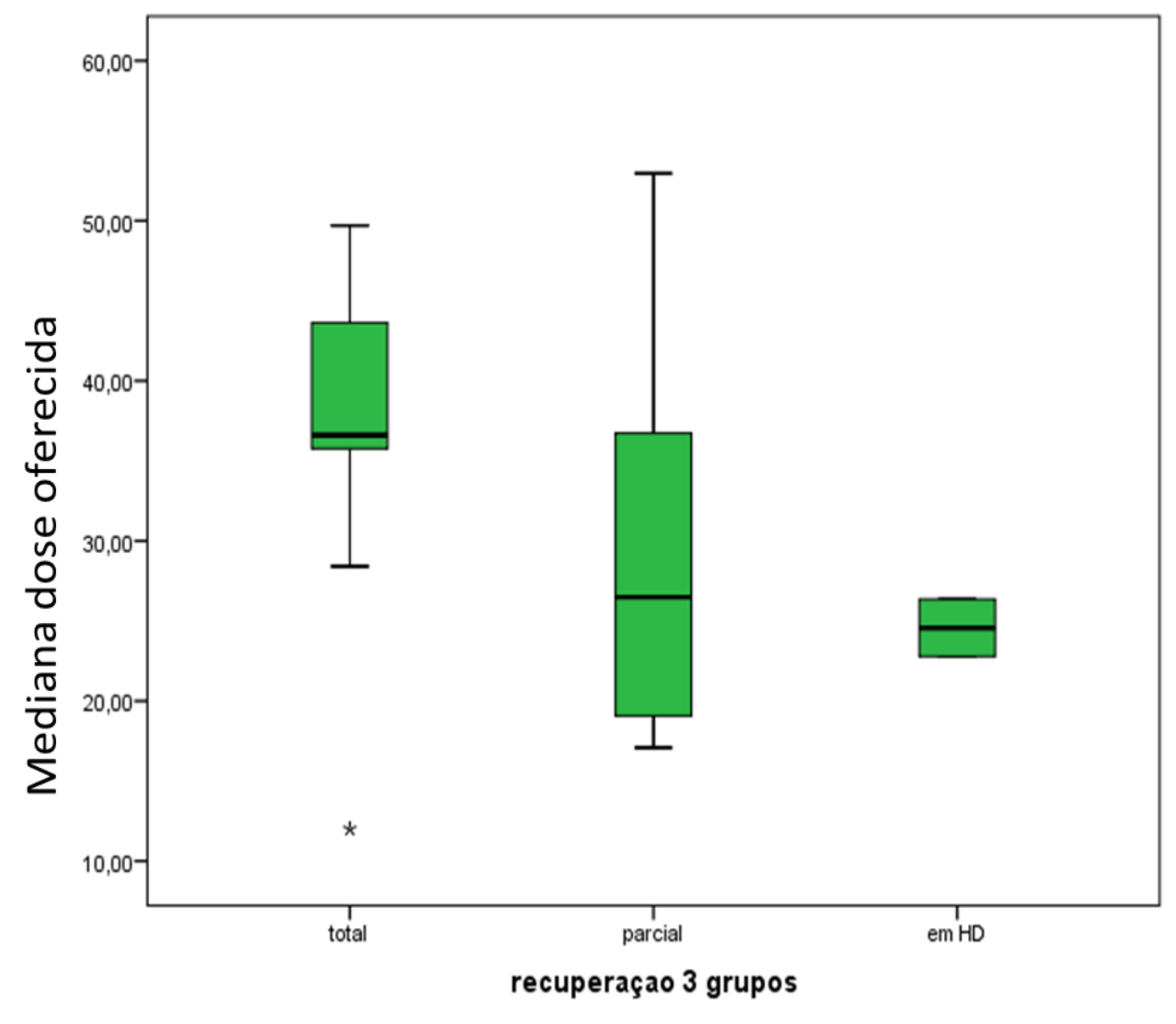

Figura 9 - Média da dose diálise durante os primeiros sete dias de TRS em relação à recuperação da função renal 
Pacientes que recuperaram a função renal total apresentaram maior tempo para atingir o controle de uréia, 3 dias vs. 2 dias, que pacientes que recuperaram a função renal parcial. Com relação ao controle acidobásico, os pacientes que recuperaram a função renal total apresentaram mediana de tempo para atingir controle do bicarbonato maior que os pacientes que recuperaram a função renal parcialmente, mas sem diferença estatística ( 3 dias vs. 2 dias, $\mathrm{p}=0,419$ ) e mediana de tempo menor para atingir o controle do $\mathrm{pH}$, também sem diferença estatística (2 dias $v s .3$ dias, $\mathrm{p}=0,781$ ).

Nos pacientes que recuperaram a função renal total o tempo para atingir $\mathrm{BH}$ zerado foi o mesmo quando comparado aos pacientes que recuperaram parcialmente a função renal: 1 dia (1-2) vs. 1 dia (1-3).

A mediana da UF quando todas as diálises foram analisadas, ou analisando separadamente mediana da UF dos métodos intermitentes e métodos contínuos, não foram diferentes em relação à recuperação da função renal $(p=0,067, p=0,363$ e $p$ $=0,244$, respectivamente) .

\subsection{Recuperação da função renal em relação ao momento de início da TRS}

Não houve diferença com relação à recuperação da função renal na alta hospitalar entre os pacientes iniciaram a TRS com uréia maior que $120 \mathrm{mg} / \mathrm{dL}$ e os que começaram com uréia menor que este valor

Os dois pacientes que permaneceram em hemodiálise iniciaram TRS com AKIN 3, contudo, não houve diferença em relação a recuperação da função renal entre os pacientes que iniciaram a diálise com AKIN 1 ou 2 ou AKIN 3. 
O menor tempo de internação na UTI no início da TRS esteve associado com uma maior taxa de recuperação da função renal total quando comparado com recuperação parcial e sem recuperação, mas sem significância estatística $(p=0,145)$. A figura 10 mostra o tempo internação na UTI no início da TRS em relação à recuperação da função renal na alta hospitalar. Dos pacientes que iniciaram TRS antes de completar dois dias de internação, $68,2 \%$ recuperaram totalmente a função renal.

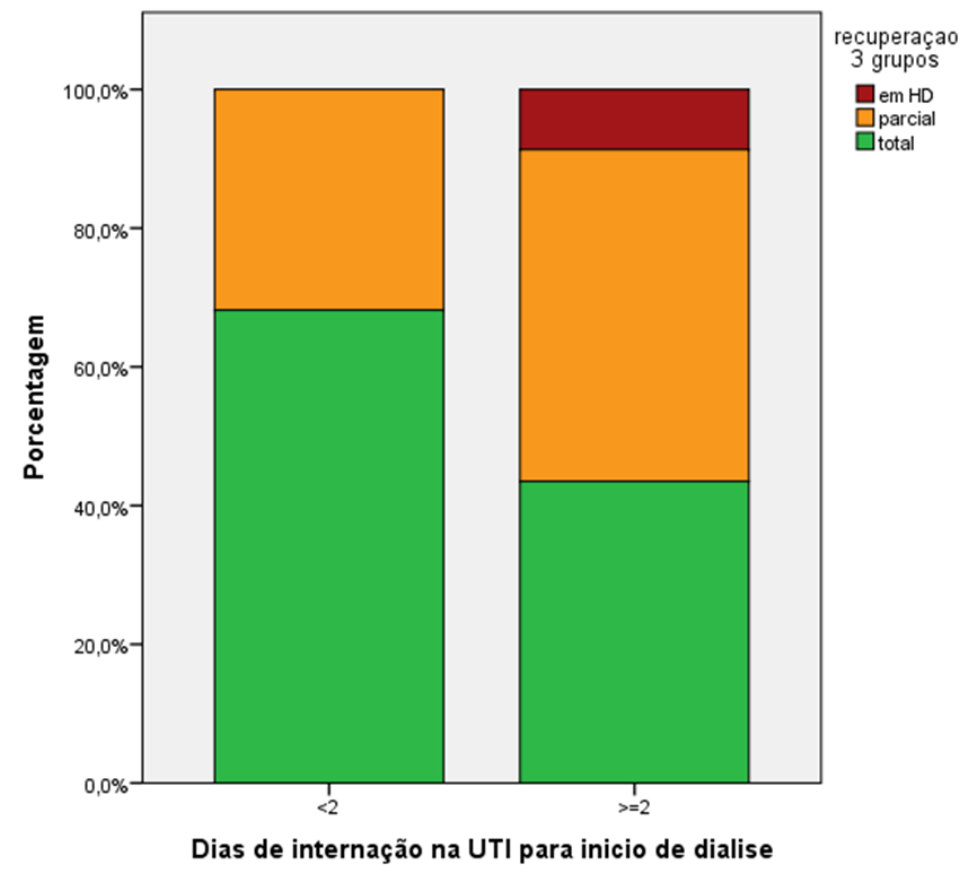

Figura 10 - Recuperação da função renal em relação ao tempo de UTI no momento de início da terapia renal 


\section{DISCUSSÃO}

Neste estudo prospectivo de coorte observacional, a mortalidade hospitalar dos pacientes com IRA dialítica foi alta, 73,2\%. O Hospital das clínicas da faculdade de medicina da USP é um centro quaternário, que recebe pacientes de alta complexidade clínica e cirúrgica. A mortalidade, apesar de ser mais elevada do que em outros centros internacionais, condiz com resultados recentes que mostram que, em pacientes com IRA dialítica, a mortalidade persiste alta (40-42), mesmo após os avanços nos cuidados intensivos e nas técnicas de diálise.

Foram avaliadas as taxas de mortalidade hospitalar em relação ao momento de início da TRS, de acordo com as diferentes definições de precocidade. Utilizamos três diferentes parâmetros para classificar momento de início da TRS como precoce ou tardio: nível de uréia, estágio do AKIN e tempo de internação em UTI no início da TRS. O nível de uréia definido como precoce foi menor que $120 \mathrm{mg} / \mathrm{dL}$ de acordo com a literatura (43). Pacientes iniciando a TRS com AKIN 1 ou 2 foram considerados como precoce, e iniciando com AKIN 3 como tardio. O tempo de internação em UTI no início da TRS menor que dois dias foi considerado como início precoce (38)

O limite de uréia para determinar início precoce é arbitrário, e varia com a época do estudo. Alguns estudos retrospectivos mostram maior mortalidade no grupo de início tardio baseado em níveis de uréia sérica $(34,35)$ enquanto outros não. Ostermann e col. (43) demonstrou em seu estudo retrospectivo, multicêntrico, com 1847 pacientes, a análise de vários parâmetros como critério para determinar início 
precoce e tardio, inclusive a uréia. Neste estudo os autores não encontraram diferença na mortalidade tanto na UTI (54\% vs. 54,2\%, p=1), quanto hospitalar. Bagshaw e col., utilizando dados do BEST ("Beginning and Ending Supportive Therapy”) (38), um estudo prospectivo, multicêntrico, com 1238 pacientes, também classificou início precoce e tardio utilizando uréia como parâmetro e também não encontraram diferença na mortalidade hospitalar (OR, 1,25;95\% IC, 0,91-1,70; $\mathrm{p}=$ $0,16)$.

No nosso estudo, utilizando a uréia como parâmetro para definir momento de início da TRS como precoce ou tardio, não foi encontrado diferença na mortalidade hospitalar entre os pacientes que começaram com uréia menor que $120 \mathrm{mg} / \mathrm{dL}$ e os que começaram com uréia maior que este valor.

A uréia é um parâmetro clássico bastante utilizado para estratificar momento de início da TRS, mas alvo de muitas críticas. A sua concentração depende do volume de distribuição, com isso, principalmente em pacientes críticos que estão sujeitos a uma maior sobrecarga de volume, a concentração sérica deste marcador pode estar mais baixa. Outro fator que contribui com os argumentos de que a uréia é um parâmetro ruim para definir precocidade, é que sua concentração depende também da taxa de produção e da reabsorção tubular renal. Pacientes críticos, devido ao alto catabolismo, podem estar com níveis elevados de uréia não refletindo a real disfunção renal. De Corte e col. (44) avaliaram se diferentes pontos de corte da concentração de uréia sérica, como recomendados na literatura, estavam associados com mortalidade hospitalar no momento de início da TRS. O estudo mostrou que, os diferentes pontos de corte da concentração da uréia para definir momento de início 
da TRS, apresentam baixa área sob a curva ROC e, portanto, baixa sensibilidade e especificidade para predição de mortalidade hospitalar.

O AKIN e o RIFLE foram introduzidos para tentar padronizar a definição e o estágio da IRA. Mas alguns estudos utilizaram os estágios da IRA para classificar o tempo de início da TRS. A vantagem destes parâmetros é que podem combinar o critério do aumento da creatinina e a queda do debito urinário, ao invés de considerar apenas um critério isolado. No entanto, alguns estudos retrospectivos, que utilizaram apenas o critério da creatinina para determinar a classe do RIFLE, por exemplo, mostraram resultados contraditórios. No trabalho de Maccariello e col. (45), um estudo brasileiro, prospectivo, multicêntrico, foram avaliados 214 pacientes, sendo que $84 \%$ realizaram diálise contínua, $25 \%$ estavam no estagio R, $27 \%$ no estagio I e $48 \%$ no estagio $\mathrm{F}$ quando iniciaram a TRS e não houve diferença na mortalidade hospitalar. No entanto, no estudo do Shiao e col (46), prospectivo e multicêntrico, 98 pacientes com IRA pós-cirurgia abdominal, o início da terapia dialítica com classificação de RIFLE I ou F (tardio), foi, independentemente, associado a maior mortalidade hospitalar (Hazard Ratio 1,84, p=0,027). Além da limitação, já mencionada, de não utilizar também o débito urinário para determinar o RIFLE, o número de pacientes deste estudo pode não ser suficiente para determinar mortalidade hospitalar.

Quando utilizamos os estágios do AKIN, tanto pelo critério da creatinina quanto pelo critério do débito urinário, para avaliar tempo de início da TRS e resultados, não houve diferença em relação à mortalidade hospitalar entre os 
pacientes que iniciaram a TRS com AKIN 1 ou 2, comparados com os pacientes que iniciaram com AKIN $3(\mathrm{p}=0,468)$.

O terceiro critério utilizado pelo presente estudo, como parâmetro de tempo de início da TRS, foi tempo de internação na UTI no início da TRS. O menor tempo de internação na UTI no início da TRS esteve associado com menor mortalidade hospitalar. Pacientes que iniciaram a TRS antes de 2 dias da internação em UTI tiveram menor mortalidade hospitalar, $62,7 \%$ vs. $73,9 \%$ quando a TRS foi iniciada entre 2 dias e 5 dias, vs. 82,5\% quando TRS iniciada após 5 dias de admissão em UTI, $\mathrm{p}=0,046$.

Como no nosso estudo, o trabalho do BEST (38), que estratificou tempo de início da TRS em precoce e tardio utilizando três parâmetros, só mostrou diferença na mortalidade hospitalar quando utilizou o critério de tempo de internação na UTI no início da TRS. Eles mostraram que início tardio da TRS esteve associado com maior mortalidade (OR 2,2; 95\% IC 1,44-3,37, $\mathrm{p}=0,001$ ).

Como já mencionado, a diferenciação entre o que é início precoce e o que é tardio é variável e se baseia em limites arbitrários dos parâmetros utilizados. A maioria das evidências em favor da TRS precoce, a exemplo deste estudo, vem de estudos observacionais. Melhores resultados com TRS precoce pode refletir a inclusão de pacientes com menor grau de lesões em órgãos alvo e/ou inclusão de pacientes com IRA transitória, que poderiam ter recuperação espontânea. Existe uma grande discussão hoje, com relação aos desfechos comparando início da TRS precoce vs. tratamento conservador. Payen e col. (47), em seu estudo prospectivo, randomizado em pacientes com sepse grave, mostrou que TRS precoce pode ser prejudicial quando comparado com tratamento conservador. Porém, estudos mais 
recentes tem demonstrado melhor sobrevida no grupo TRS (33). Vaara e col. (48) realizaram um estudo com os dados do FINNAKI (Finnish Acute Kidney Injury) Study Group. Eles mostraram que, os pacientes que iniciaram TRS na ausência de qualquer indicador convencional (grupo que eles chamaram de pré-emptivo), apresentaram mortalidade em 90 dias menor quando comparados tanto com o grupo que iniciou terapia dialítica na presença de qualquer indicador convencional, quanto com o grupo que não realizou TRS. Também, Wilson e col. (49) em estudo recente, mostrou que TRS está associado a uma melhor sobrevida apenas em pacientes com um elevado nível de creatinina sérica $(>4.2 \mathrm{mg} / \mathrm{dL})$. No entanto, este estudo não inclui todas as complicações da IRA e o débito urinário como variáveis ajustadas.

Novos parâmetros de início da TRS são propostos para avaliar desfechos, e balanço hídrico é um deles. Balanço hídrico $(\mathrm{BH})$ é um parâmetro importante porque sobrecarga hídrica é frequente em pacientes críticos, ocorrendo em 30 a 70\%. No presente estudo, quando avaliamos o $\mathrm{BH}$ acumulado dos 3 dias antes até o início da TRS, nos pacientes que evoluíram para óbito, o acúmulo de fluidos foi significativamente maior comparado aos sobreviventes $(3090 \mathrm{ml}$ vs. $1632 \mathrm{ml}$, $\mathrm{p}=0,004)$. O comportamento do $\mathrm{BH}$ ao longo dos dias não foi diferente entre os sobreviventes e não sobreviventes, que apresentaram o mesmo tempo para atingir o BH zerado após o início da TRS ( 1 dia vs. 1 dia, $\mathrm{p}=0,750$ ).

A porcentagem de pacientes que evoluiu para óbito foi maior, quanto maior a porcentagem de $\mathrm{BH}$ em relação ao peso. Resultado semelhante ao encontrado no estudo da Bouchard e col. (50), uma análise de dados do PICARD, que, além de mostrar maior sobrevida nos pacientes que apresentavam $\mathrm{BH}$ menor no início da diálise, mostra também um aumento gradual da mortalidade quanto maior é a 
sobrecarga hídrica. Fülop, T e col. (51) também mostra associação de sobrecarga hídrica com mortalidade. Neste estudo o Odds Ratio (OR) para mortalidade na análise multivariada foi 2,62 (95\% IC: 1,07-6,44) quando a porcentagem de ganho de peso $\geq 10 \%$ e OR de 3,98 (95\% CI: $1,01-15,75)$ quando apresentaram porcentagem de ganho de peso $\geq 20 \%(p=0,049)$. Embora a associação entre $\mathrm{BH}$ positivo e mortalidade pareça óbvia, não existe ainda uma relação de causa e efeito definitiva já que a maioria dos trabalhos é observacional.

Além do momento de início da TRS, a dose de diálise necessária para melhorar desfechos permanece em discussão. Este estudo também avaliou a dose de diálise em relação à mortalidade hospitalar.

Os pacientes não sobreviventes tiveram mediana de dose de diálise e Kt/V discretamente menores quando comparados aos sobreviventes $(35,1 \mathrm{ml} / \mathrm{kg} / \mathrm{h}$ vs. 35,7 $\mathrm{ml} / \mathrm{kg} / \mathrm{h}$ e 1,47 vs. 1,50 respectivamente), sem significância estatística. Os dois últimos grandes estudos multicêntricos que avaliaram dose de diálise $(21,22)$, não conseguiram mostrar benefício na sobrevida com uma maior dose de diálise.

Na análise multivariada para avaliar mortalidade hospitalar incluindo idade, tipo de internação, hipoalbuminemia, HAS, câncer prévio, DRC, sedação, droga vasoativa e tempo de internação na UTI no início da TRS. As variáveis, idade, ausência de sedação e tempo de internação na UTI no início da TRS estiveram associados à mortalidade hospitalar. O maior tempo para início da TRS em relação à admissão na UTI manteve associação, de forma independente, com maior mortalidade hospitalar (OR 4,70; 95\% IC 1,0-21,9 para os pacientes que começaram entre 2-5 dias e OR 20,93; IC 3,1-139,3 para os pacientes que começaram após 5 dias de admissão na UTI), assim como a idade (OR 1,05; 95\% IC 1,0 - 1,1). 
Outro parâmetro independentemente associados com mortalidade hospitalar foi sedação. A ausência de sedação foi associada com uma menor mortalidade (OR 0,10; IC 95\% 0,02-0,50). Embora a sedação possa apenas refletir a gravidade da doença, este parâmetro pode ser um alvo para futuros estudos. A necessidade de sedação e os medicamentos utilizados pode ter influência na gravidade e mortalidade em pacientes críticos (52).

Com relação à recuperação da função renal, não houve associação com dose e com tempo de início da TRS utilizando os três parâmetros para definir precocidade: uréia, estágio do AKIN e tempo de internação em UTI no início da TRS.

Nosso estudo traçou o perfil dos pacientes críticos internados em UTI com IRA submetidos à TRS de um hospital terciário. Os resultados de nossa coorte, em relação à influência do tempo de início e da dose de diálise, na evolução dos pacientes com IRA em UTI são comparáveis às grandes coortes descritas na literatura.

Nosso estudo apresenta algumas limitações. É um estudo observacional e utilizamos amostra de conveniência em uma única instituição hospitalar. Por outro lado, nossos achados ressaltam as limitações do uso de parâmetros atualmente utilizados como critério para definição de início precoce e tardio.

O uso de novos biomarcadores além de poderem resultar em avanços no diagnóstico e estadiamento da IRA, poderia também ser utilizado para avaliar se intervenções mais precoces, são capazes de reduzir a elevada mortalidade associada à IRA.

Até o momento, também não está bem estabelecido o melhor modo de avaliar dose de diálise nos pacientes críticos. Nossos achados também reafirmam que os 
parâmetros atualmente utilizados para avaliar dose de diálise, $\mathrm{Kt} / \mathrm{V}$ para os métodos intermitentes e taxa de efluentes para terapias contínuas, podem não ser o melhor modo para essa avaliação. Outros parâmetros de avaliação da dose deveriam ser considerados, como controle metabólico e acido básico e controle do balanço hídrico. 


\section{CONCLUSÃO}

Este estudo de coorte observacional fez uma análise retrospectiva, de uma base de dados prospectiva, do desfecho mortalidade hospitalar com relação a momento de início da TRS e dose de diálise. O início precoce baseado em dados laboratoriais relacionados à gravidade da IRA, nível de uréia e o estágio da IRA baseado na classificação de AKIN, no início da TRS, não foram associados com a mortalidade hospitalar. O início precoce da TRS em relação ao tempo de admissão na UTI esteve independentemente associado a menor mortalidade hospitalar. A dose de diálise não teve associação com mortalidade hospitalar. $O$ atraso no reconhecimento da gravidade da IRA, assim como para a instituição do tratamento dialítico, pode ter impacto na evolução dos pacientes com IRA. Estudos futuros devem avaliar se o início precoce baseado em parâmetros temporais pode ser um fator que altere a mortalidade associada à IRA dialítica. 


\section{ANEXOS}

\subsection{ANEXO A}

SOFA Escore ${ }^{5}$

\begin{tabular}{|c|c|c|c|c|c|}
\hline SOFA Escore & 0 & 1 & 2 & 3 & 4 \\
\hline \multicolumn{6}{|l|}{ Respiração } \\
\hline $\mathrm{PaO2} / \mathrm{FiO2}$ (a) & $>400$ & $<400$ & $<300$ & $<200$ (a) & $<100$ \\
\hline \multicolumn{6}{|l|}{ Coagulação } \\
\hline Plaquetas $10^{3} / \mathrm{mm}^{3}$ & $>150$ & $<150$ & $<100$ & $<50$ & $<20$ \\
\hline Hipotensão & & & Dopamina $\leq 5$ & Dopamina $>5$ & Dopamina $>15$ \\
\hline Cardiovascular (b) & $\mathrm{PAM}>70$ & $\mathrm{PAM}<70$ & $\begin{array}{l}\text { ou Dobutamina, } \\
\text { qualquer dose }\end{array}$ & $\begin{array}{l}\text { ou Epinefrina } \leq 0.1 \\
\text { ou Norepinefrina } \leq 0.1\end{array}$ & $\begin{array}{l}\text { ou Epinefrina }>0.1 \\
\text { ou Norepinefrina }>0.1\end{array}$ \\
\hline \multicolumn{6}{|l|}{ Fígado } \\
\hline bilirrubina mg/dl & $<1.2$ & $1.2-1.9$ & $2.0-5.9$ & $6.0-11.9$ & $>12.0$ \\
\hline $\begin{array}{l}\text { SNC escala de coma } \\
\text { de Glasgow }\end{array}$ & $>14$ & $13 \cdot 14$ & $10 \cdot 12$ & $6-9$ & $<6$ \\
\hline $\begin{array}{l}\text { Renal creatinina } \\
\text { ou débito urinário }\end{array}$ & $<1.2$ & $1.2-1.9$ & $2.0-3.4$ & $3.5-4.9<500$ & $>5$ ou $<200$ \\
\hline
\end{tabular}

Sampaio, FBA. Revista da SOCERJ - Mar/Abr 2005 


\subsection{ANEXO B}

\section{SAPS III ESCORE}

\begin{tabular}{|c|c|c|c|c|c|}
\hline \multicolumn{2}{|c|}{ Demográfico / estado prévio de saúde } & \multicolumn{2}{|l|}{ Categoria diagnóstica } & \multicolumn{2}{|c|}{ Variáveis fisiológicas na admissão } \\
\hline Variáveis & Pontos & Variáveis & Pontos & Variáveis & Pontos \\
\hline Idade & & Admissão programada & 0 & Glasgow & \\
\hline$<40$ & 0 & Admissão não programada & 3 & $3-4$ & 15 \\
\hline$\geq 40-<60$ & 5 & Urgência & & 5 & 10 \\
\hline$\geq 60-<70$ & 9 & Não cirúrgico & 5 & 6 & 7 \\
\hline$\geq 70-<75$ & 13 & Eletiva & 0 & $7-12$ & 2 \\
\hline$\geq 75-<80$ & 15 & Emergência & 6 & $\geq 13$ & 0 \\
\hline$\geq 80$ & 18 & Tipo de operação & & Frequência cardiaca & \\
\hline Comorbidades & & Transplantes & -11 & $<120$ & 0 \\
\hline Outras & 0 & Trauma & -8 & $\geq 120-<160$ & 5 \\
\hline Quimioterapia & 3 & RM sem valva & -6 & $\geq 160$ & 7 \\
\hline ICC NYHA IV & 6 & Cirurgia no AVC & 5 & Pressão arterial sistólica & \\
\hline Neoplasia hematológica & 6 & Outras & 0 & $<40$ & 11 \\
\hline Cirrose & 8 & Admissão na UTI acrescentar 16 pontos & 16 & $\geq 40-<70$ & 8 \\
\hline Aids & 8 & Motivo de internação & & $\geq 70-<120$ & 3 \\
\hline Metástase & 11 & Neurológicas & & $\geq 120$ & 0 \\
\hline Dias de internação prévios & & Convulsões & -4 & Oxigenação & \\
\hline$<14$ & 0 & Coma, confusão, agitação & 4 & VM relação $\mathrm{PaO}_{2} / \mathrm{FiO}_{2}<100$ & 11 \\
\hline$\geq 14-28$ & 6 & Déficit Focal & 7 & VM relação $\geq 100$ & 7 \\
\hline$\geq 28$ & 7 & Efeito de massa intracraniana & 11 & Sem VM $\mathrm{PaO}_{2}<60$ & 5 \\
\hline Procedência & & Cardiológicas & & Sem VM $\mathrm{PaO}_{2} \geq 60$ & 0 \\
\hline Centro cirúrgico & 0 & Arritmia & -5 & Temperatura & \\
\hline PS & 5 & Choque hemorrágico & 3 & $<34,5$ & 7 \\
\hline Outra UTI & 7 & Choque hipovolêmico não hemorrágico & 3 & $\geq 34,5$ & 0 \\
\hline Outros & 8 & Choque distributivo & 5 & Leucócitos & \\
\hline Fármacos vasoativos & & Abdômen & & $<15.000$ & 0 \\
\hline Sim & 0 & Abdômen agudo & 3 & $\geq 15.000$ & 2 \\
\hline Não & 3 & Pancreatite grave & 9 & Plaquetas & \\
\hline & & Falência hepática & 6 & $<20.000$ & 13 \\
\hline & & Outras & 0 & $\geq 20.000-<50.000$ & 8 \\
\hline & & Infecção & & $\geq 50.000-<100.000$ & 5 \\
\hline & & Nosocomial & 4 & $\geq 100.000$ & 0 \\
\hline & & Respiratória & 5 & $\mathrm{pH}$ & \\
\hline & & Outras & 0 & $\leq 7,25$ & 3 \\
\hline & & & & $>7,25$ & 0 \\
\hline & & & & Creatinina & \\
\hline & & & & $<1,2$ & 0 \\
\hline & & & & $\geq 1,2-<2,0$ & 2 \\
\hline & & & & $\geq 2,0-<3,5$ & 7 \\
\hline & & & & $\geq 3,5$ & 8 \\
\hline & & & & Bilirrubina & \\
\hline & & & & $<2$ & 0 \\
\hline & & & & $\geq 2-<6$ & 4 \\
\hline & & & & $\geq 6$ & 5 \\
\hline Total & & & & & \\
\hline
\end{tabular}

Adaptado de Moreno RP. Intensive Care Med 2005; 31: 1345-55. 


\section{REFERÊNCIAS}

1. Waikar SS, Curhan GC, Wald R, McCarthy EP, Chertow GM. Declining mortality in patients with acute renal failure, 1988 to 2002 . J Am Soc Nephrol. 2006;17(4):1143-50.

2. Xue JL, Daniels F, Star RA, Kimmel PL, Eggers PW, Molitoris BA, et al. Incidence and mortality of acute renal failure in Medicare beneficiaries, 1992 to 2001. J Am Soc Nephrol. 2006;17(4):1135-42.

3. Bagshaw SM, George C, Bellomo R. Changes in the incidence and outcome for early acute kidney injury in a cohort of Australian intensive care units. Crit Care. 2007;11(3):R68.

4. Hsu CY, McCulloch CE, Fan D, Ordoñez JD, Chertow GM, Go AS. Community-based incidence of acute renal failure. Kidney Int. 2007;72(2):208-12.

5. Cosentino F, Chaff C, Piedmonte M. Risk factors influencing survival in ICU acute renal failure. Nephrol Dial Transplant. 1994;9 Suppl 4:179-82.

6. Liaño F, Pascual J. Epidemiology of acute renal failure: a prospective, multicenter, community-based study. Madrid Acute Renal Failure Study Group. Kidney Int. 1996;50(3):811-8.

7. Levy EM, Viscoli CM, Horwitz RI. The effect of acute renal failure on mortality. A cohort analysis. JAMA. 1996;275(19):1489-94.

8. Bellomo R, Ronco C, Kellum JA, Mehta RL, Palevsky P. Acute renal failure - definition, outcome measures, animal models, fluid therapy and information technology needs: the Second International Consensus Conference of the Acute Dialysis Quality Initiative (ADQI) Group. Crit Care. 2004;8(4):R204-12.

9. Mehta R, Kellum J, Shah S, Molitoris B, Ronco C, Warnock D, et al. Acute Kidney Injury Network: report of an initiative to improve outcomes in acute kidney injury. Crit Care. 2007;11(2):R31.

10. Ricci Z, Cruz D, Ronco C. The RIFLE criteria and mortality in acute kidney injury: A systematic review. Kidney Int. 2008;73(5):538-46.

11. Hoste EA, Schurgers M. Epidemiology of acute kidney injury: how big is the problem? Crit Care Med. 2008;36(4 Suppl):S146-51.

12. Srisawat N, Hoste EE, Kellum JA. Modern classification of acute kidney injury. Blood Purif.29(3):300-7.

13. Kidney Disease: Improving Global

Outcomes (KDIGO) Acute Kidney Injury Work Group. KDIGO Clinical Practice Guideline

for Acute Kidney Injury. Kidney inter., Suppl. 2012; 2: 1-138.

14. Harter HR. Review of significant findings from the National Cooperative Dialysis Study and recommendations. Kidney Int Suppl. 1983(13):S107-12.

15. Lowrie EG, Laird NM, Henry RR. Protocol for the National Cooperative Dialysis Study. Kidney Int Suppl. 1983(13):S11-8.

16. Evanson JA, Ikizler TA, Wingard R, Knights S, Shyr Y, Schulman G, et al. Measurement of the delivery of dialysis in acute renal failure. Kidney Int. 1999;55(4):1501-8. 
17. Gillum DM, Dixon BS, Yanover MJ, Kelleher SP, Shapiro MD, Benedetti RG, et al. The role of intensive dialysis in acute renal failure. Clin Nephrol. 1986;25(5):249-55.

18. Paganini EP, Halstenberg WK, Goormastic M. Risk modeling in acute renal failure requiring dialysis: the introduction of a new model. Clin Nephrol. 1996;46(3):206-11.

19. Ronco C, Bellomo R, Homel P, Brendolan A, Dan M, Piccinni P, et al. Effects of different doses in continuous veno-venous haemofiltration on outcomes of acute renal failure: a prospective randomised trial. Lancet. 2000;356(9223):26-30.

20. Schiffl H, Lang SM, Fischer R. Daily hemodialysis and the outcome of acute renal failure. N Engl J Med. 2002;346(5):305-10.

21. Palevsky PM, Zhang JH, O'Connor TZ, Chertow GM, Crowley ST, Choudhury $D$, et al. Intensity of renal support in critically ill patients with acute kidney injury. N Engl J Med. 2008;359(1):7-20.

22. Bellomo R, Cass A, Cole L, Finfer S, Gallagher M, Lo S, et al. Intensity of continuous renal-replacement therapy in critically ill patients. $\mathrm{N}$ Engl $\mathrm{J}$ Med. 2009;361(17):1627-38.

23. Karvellas CJ, Farhat MR, Sajjad I, Mogensen SS, Leung AA, Wald R, et al. A comparison of early versus late initiation of renal replacement therapy in critically ill patients with acute kidney injury: a systematic review and metaanalysis. Crit Care. 2011;15(1):R72.

24. Mehta RL. Indications for dialysis in the ICU: renal replacement vs. renal support. Blood Purif. 2001;19(2):227-32.

25. Ricci Z, Ronco C. Timing, dose and mode of dialysis in acute kidney injury. Curr Opin Crit Care. 2011;17(6):556-61.

26. Palevsky PM. Renal replacement therapy I: indications and timing. Crit Care Clin. 2005;21(2):347-56.

27. Granata A, D'Intini V, Bellomo R, Ronco C. Vascular access for acute extracorporeal renal replacement therapies. Contrib Nephrol. 2004;142:15977.

28. Clermont G, Acker CG, Angus DC, Sirio CA, Pinsky MR, Johnson JP. Renal failure in the ICU: comparison of the impact of acute renal failure and end-stage renal disease on ICU outcomes. Kidney Int. 2002;62(3):986-96.

29. Mehta RL, Pascual MT, Soroko S, Savage BR, Himmelfarb J, Ikizler TA, et al. Spectrum of acute renal failure in the intensive care unit: the PICARD experience. Kidney Int. 2004;66(4):1613-21.

30. Elseviers MM, Lins RL, Van der Niepen P, Hoste E, Malbrain ML, Damas $P$, et al. Renal replacement therapy is an independent risk factor for mortality in critically ill patients with acute kidney injury. Crit Care. 2010;14(6):R221.

31. Clec'h C, Gonzalez F, Lautrette A, Nguile-Makao M, GarrousteOrgeas $\mathrm{M}$, Jamali $\mathrm{S}$, et al. Multiple-center evaluation of mortality associated with acute kidney injury in critically ill patients: a competing risks analysis. Crit Care. 2011;15(3):R128.

32. Clec'h C, Darmon M, Lautrette A, Chemouni F, Azoulay E, Schwebel C, et al. Efficacy of renal replacement therapy in critically ill patients: a propensity analysis. Crit Care. 2012;16(6):R236. 
33. Libório AB, Leite TT, Neves FM, Teles F, Bezerra CT. AKI Complications in Critically III Patients: Association with Mortality Rates and RRT. Clin J Am Soc Nephrol. 2014.

34. Gettings LG, Reynolds HN, Scalea T. Outcome in post-traumatic acute renal failure when continuous renal replacement therapy is applied early vs. late. Intensive Care Med. 1999;25(8):805-13.

35. Liu KD, Himmelfarb J, Paganini E, Ikizler TA, Soroko SH, Mehta RL, et al. Timing of initiation of dialysis in critically ill patients with acute kidney injury. Clin J Am Soc Nephrol. 2006;1(5):915-9.

36. Saudan P, Niederberger M, De Seigneux S, Romand J, Pugin J, Perneger $\mathrm{T}$, et al. Adding a dialysis dose to continuous hemofiltration increases survival in patients with acute renal failure. Kidney Int. 2006;70(7):1312-7.

37. Bouman CS, Oudemans-Van Straaten HM, Tijssen JG, Zandstra DF, Kesecioglu J. Effects of early high-volume continuous venovenous hemofiltration on survival and recovery of renal function in intensive care patients with acute renal failure: a prospective, randomized trial. Crit Care Med. 2002;30(10):2205-11.

38. Bagshaw SM, Uchino S, Bellomo R, Morimatsu H, Morgera S, Schetz $\mathrm{M}$, et al. Timing of renal replacement therapy and clinical outcomes in critically ill patients with severe acute kidney injury. $J$ Crit Care. 2009;24(1):129-40.

39. Levey AS, Bosch JP, Lewis JB, Greene T, Rogers N, Roth D. A more accurate method to estimate glomerular filtration rate from serum creatinine: a new prediction equation. Modification of Diet in Renal Disease Study Group. Ann Intern Med. 1999;130(6):461-70.

40. Brivet FG, Kleinknecht DJ, Loirat $P$, Landais PJ. Acute renal failure in intensive care units--causes, outcome, and prognostic factors of hospital mortality; a prospective, multicenter study. French Study Group on Acute Renal Failure. Crit Care Med. 1996;24(2):192-8.

41. Liaño F, Junco E, Pascual J, Madero R, Verde E. The spectrum of acute renal failure in the intensive care unit compared with that seen in other settings. The Madrid Acute Renal Failure Study Group. Kidney Int Suppl. 1998;66:S16-24.

42. Metnitz PG, Krenn CG, Steltzer H, Lang T, Ploder J, Lenz K, et al. Effect of acute renal failure requiring renal replacement therapy on outcome in critically ill patients. Crit Care Med. 2002;30(9):2051-8.

43. Ostermann $\mathrm{M}$, Chang RW. Correlation between parameters at initiation of renal replacement therapy and outcome in patients with acute kidney injury. Crit Care. 2009;13(6):R175.

44. De Corte W, Vanholder R, Dhondt AW, De Waele JJ, Decruyenaere J, Danneels C, et al. Serum urea concentration is probably not related to outcome in ICU patients with $\mathrm{AKI}$ and renal replacement therapy. Nephrol Dial Transplant. 2011;26(10):3211-8.

45. Maccariello E, Soares M, Valente C, Nogueira L, Valença RV, Machado JE, et al. RIFLE classification in patients with acute kidney injury in need of renal replacement therapy. Intensive Care Med. 2007;33(4):597-605. 
46. Shiao CC, Wu VC, Li WY, Lin YF, Hu FC, Young GH, et al. Late initiation of renal replacement therapy is associated with worse outcomes in acute kidney injury after major abdominal surgery. Crit Care. 2009;13(5):R171.

47. Payen D, Mateo J, Cavaillon JM, Fraisse F, Floriot C, Vicaut E, et al. Impact of continuous venovenous hemofiltration on organ failure during the early phase of severe sepsis: a randomized controlled trial. Crit Care Med. 2009;37(3):803-10.

48. Vaara ST, Reinikainen M, Wald R, Bagshaw SM, Pettilä V, Group FS. Timing of RRT based on the presence of conventional indications. Clin J Am Soc Nephrol. 2014;9(9):1577-85.

49. Wilson FP, Yang W, Machado CA, Mariani LH, Borovskiy Y, Berns JS, et al. Dialysis versus nondialysis in patients with $\mathrm{AKI}$ : a propensity-matched cohort study. Clin J Am Soc Nephrol. 2014;9(4):673-81.

50. Bouchard J, Soroko SB, Chertow GM, Himmelfarb J, Ikizler TA, Paganini EP, et al. Fluid accumulation, survival and recovery of kidney function in critically ill patients with acute kidney injury. Kidney Int. 2009;76(4):422-7.

51. Fülöp T, Pathak MB, Schmidt DW, Lengvárszky Z, Juncos JP, Lebrun $\mathrm{CJ}$, et al. Volume-related weight gain and subsequent mortality in acute renal failure patients treated with continuous renal replacement therapy. ASAIO J. 2010;56(4):333-7.

52. Strøm T, Johansen RR, Prahl JO, Toft P. Sedation and renal impairment in critically ill patients: a post hoc analysis of a randomized trial. Crit Care. 2011;15(3):R119. 